\title{
Generalization of a Transformation Formula Due to Kummer and Ramanujan With Applications
}

\author{
Medhat A. Rakha ${ }^{1}$, Arjun K. Rathie ${ }^{2}$ \& Adel K. Ibrahim ${ }^{3}$ \\ ${ }^{1}$ Department of Mathematics and Statistics, College of Science, Sultan Qaboos University, Muscat, Oman \\ 2 Department of Mathematics, School of Mathematical and Physical Sciences, Centeral University of Kerala, \\ Riverside Transit Campus, Padennakkad P. O. Nileshwar, Kasaragod, Kerala, India \\ ${ }^{3}$ Mathematics Department, College of Science, Jazan University, Jazan, Saudi Arabia \\ Correspondence: Medhat A. Rakha, Department of Mathematics and Statistics, College of Science, Sultan Qaboos \\ University, P. O. Box 36, Al-khoud 123, Muscat, Oman. Tel: 968-99-640-521. E-mail: medhat@ squ.edu.om
}

Received: May 28, 2014 Accepted: June 25, 2014 Online Published: July 7, 2014

doi:10.5539/jmr.v6n3p62 URL: http://dx.doi.org/10.5539/jmr.v6n3p62

The research is financed by the research grant (05/4/33) funded by Jazan University, Jazan, Saudi Arabia

\section{Abstract}

The aim of this research paper is to find the explicit expressions of

$$
{ }_{2} F_{1}\left[\begin{array}{ccc}
a, & b ; & \\
\frac{1}{2}(a+b+i+1) & & \frac{1+x}{2}
\end{array}\right]
$$

for $i=0, \pm 1, \ldots, \pm 9$.

For $i=0$, we have the well known, interesting and useful formula due to Kummer which was independently discovered by Ramanujan. The results are derived with the help of generalizations of Gauss's second summation theorem obtained recently by Rakha et al..

As applications, we also obtained a large number of interesting results closely related to other results of Ramanujan. In the end, using Beta integral method, a large number of new and interesting hypergeometric identities are established. Known results earlier obtained by Choi et al. follow special cases of our main findings.

Keywords: hypergeometric series, Ramanujan's identities

\section{Introduction}

In 1812, C. F. Gauss (1813) systematically discussed the series

$$
\sum_{n=0}^{\infty} \frac{(a)_{n}(b)_{n}}{(c)_{n}} \frac{z^{n}}{n !}
$$

where $(a)_{n}=a(a+1) \ldots(a+n-1) ;(a)_{0}=1$, which is of fundamental importance in the theory of special functions. The series is known as the Gauss series and it is represented by the $\operatorname{symbol}_{2} F_{1}[a, b ; c ; x]$ or ${ }_{2} F_{1}\left[\begin{array}{r}a, b ; \\ c ;\end{array}\right]$.

A natural generalization of ${ }_{2} F_{1}$ is the generalized hypergeometric function, the so-called ${ }_{p} F_{q}$, which is defined in the following manner (Rainville, 1971)

$$
{ }_{p} F_{q}\left[(a)_{p} ;(b)_{q} ; z\right] \equiv{ }_{p} F_{q}\left[\begin{array}{c}
(a)_{p} ; \\
(b)_{q} ;
\end{array}\right] \equiv{ }_{p} F_{q}\left[\begin{array}{c}
a_{1}, \ldots, a_{q} ; \\
b_{1}, \ldots, b_{q} ;
\end{array}\right]=\sum_{n=0}^{\infty} \frac{\prod_{j=1}^{p}\left(a_{j}\right)_{n}}{\prod_{j=1}^{q}\left(b_{j}\right)_{n}} \frac{z^{n}}{n !} .
$$


The series on the right-hand side of (2) is absolutely convergent for all values of $z$, real or complex, when $p \leq q$. Further, when $p=q+1$, the series is convergent if $|z|<1$. It converges when $z=1$ if

$$
\operatorname{Re}\left[\sum_{j=1}^{q} b_{j}-\sum_{j=1}^{p} a_{j}\right]>0
$$

and when $|z|<1, z \neq 1$, if

$$
\operatorname{Re}\left[\sum_{j=1}^{q} b_{j}-\sum_{j=1}^{p} a_{j}\right]>-1 .
$$

If $p>q+1$, the series never converges except that $z=0$, and the function is only defined when the series terminates.

Whenever hypergeometric or generalized hypergeometric functions reduce to Gamma functions, the results are very important from the applications point of view. Thus, the classical summation theorems such as those of Gauss, Gauss second, Kummer, and Bailey for the series ${ }_{2} F_{1}$; Watson, Dixon, Whipple and Saalschütz for the series ${ }_{3} F_{2}$ and others play an important role in the theory of generalized hypergeometric series. Applications of the above mentioned classical summation theorems are well known now.

Recently good deal of progress has been done in the direction of generalizing and extending the above mentioned classical summation theorems. For this, we refer the papers by Kim et al. (2010) and Rakha and Rathie (2011).

In our present investigation, we are interested in mentioning the following Gauss's second summation theorem (Bailey, 1964) viz.

$$
{ }_{2} F_{1}\left[\begin{array}{ccc}
a, & b ; & \\
\frac{1}{2}(a+b+1) ; & & \frac{1}{2}
\end{array}\right]=\frac{\Gamma\left(\frac{1}{2}\right) \Gamma\left(\frac{1}{2} a+\frac{1}{2} b+\frac{1}{2}\right)}{\Gamma\left(\frac{1}{2} a+\frac{1}{2}\right) \Gamma\left(\frac{1}{2} b+\frac{1}{2}\right)}
$$

and the Euler's first transformation formula (Bailey, 1964) viz.

$$
{ }_{2} F_{1}\left[\begin{array}{rrr}
a, & b ; & \\
c ; & & z
\end{array}\right]=(1-z)_{2}^{-a} F_{1}\left[\begin{array}{lll}
a, & c-b ; & \\
c ; & & \frac{z}{z-1}
\end{array}\right] .
$$

Also, we mention here a transformation formula due to Kummer (1836) viz.

$$
\begin{aligned}
& { }_{2} F_{1}\left[\begin{array}{ccc}
a, & b ; & \\
\frac{1}{2}(a+b+1) ; & & \frac{1+x}{2}
\end{array}\right]=\frac{\Gamma\left(\frac{1}{2}\right) \Gamma\left(\frac{1}{2} a+\frac{1}{2} b+\frac{1}{2}\right)}{\Gamma\left(\frac{1}{2} a+\frac{1}{2}\right) \Gamma\left(\frac{1}{2} b+\frac{1}{2}\right)}{ }_{2} F_{1}\left[\begin{array}{ccc}
\frac{1}{2} a, & \frac{1}{2} b ; & \\
\frac{1}{2} ; & & x^{2}
\end{array}\right] \\
& +\frac{2 x \Gamma\left(\frac{1}{2}\right) \Gamma\left(\frac{1}{2} a+\frac{1}{2} b+\frac{1}{2}\right)}{\Gamma\left(\frac{1}{2} a\right) \Gamma\left(\frac{1}{2} b\right)}{ }_{2} F_{1}\left[\begin{array}{ccc}
\frac{1}{2} a+\frac{1}{2}, & \frac{1}{2} b+\frac{1}{2} ; \\
\frac{3}{2} ; & x^{2}
\end{array}\right] .
\end{aligned}
$$

It is not out of place to mention here that the formula (5) was also independently rediscovered by Ramanujan (Berndt, 1989, Entry 21, p. 64).

Further in (5), if we take $a=b=\frac{1}{2}$, we get the following another result due to Ramanujan (Berndt, 1989, p. 96)

$$
{ }_{2} F_{1}\left[\begin{array}{ccc}
\frac{1}{2}, & \frac{1}{2} ; & \\
1 ; & \frac{1+x}{2}
\end{array}\right]=\mu_{2} F_{1}\left[\begin{array}{lll}
\frac{1}{4}, & \frac{1}{4} ; \\
\frac{1}{2} ; & & x^{2}
\end{array}\right]+\eta x_{2} F_{1}\left[\begin{array}{lll}
\frac{3}{4}, & \frac{3}{4} ; & \\
\frac{3}{2} ; & &
\end{array}\right] .
$$

where $\mu$ and $\eta$ are defined by

$$
\mu=\frac{\Gamma\left(\frac{1}{2}\right)}{\left\{\Gamma\left(\frac{3}{4}\right)\right\}^{2}} \text { and } \eta=\frac{\left\{\Gamma\left(\frac{3}{4}\right)\right\}^{2}}{\left\{\Gamma\left(\frac{1}{2}\right)\right\}^{3}} .
$$

The result (6) was derived by Berndt (1989) by utilizing Gauss's second summation Theorem (3). Further, in (6), if we replace $x$ by $\frac{2 x}{1+x^{2}}$ and using the following result due to Gauss (Berndt, 1989, p. 2) (which is also obtained 
independently by Ramanujan (Berndt, 1989, Entry 3, p. 50) viz.

$$
{ }_{2} F_{1}\left[\begin{array}{crr}
r, & m ; & \\
2 m ; & & \frac{4 x}{(1+x)^{2}}
\end{array}\right]=(1+x)^{2 r}{ }_{2} F_{1}\left[\begin{array}{ccc}
r, & r-m+\frac{1}{2} ; & \\
m+\frac{1}{2} ; & & x^{2}
\end{array}\right]
$$

twice (for (i) $r=m=\frac{1}{4}$ and (ii) $r=m=\frac{3}{4}$ ), we get the following corrected result due to Ramanujan (Rakha et al., 2013) viz:

$$
{ }_{2} F_{1}\left[\begin{array}{lll}
\frac{1}{2}, & \frac{1}{2} ; \\
1 ; & \frac{1}{2}+\frac{x}{1+x^{2}}
\end{array}\right]=\mu \sqrt{1+x^{2}}{ }_{2} F_{1}\left[\begin{array}{ll}
\frac{1}{4}, & \frac{1}{2} ; \\
\frac{3}{4} ; &
\end{array}\right]+2 \eta x \sqrt{1+x^{2}}{ }_{2} F_{1}\left[\begin{array}{lll}
\frac{3}{4}, & \frac{1}{2} ; & \\
\frac{5}{4} ; &
\end{array}\right] .
$$

Finally, applying Euler's transformation formula (4) in the right-hand side of (9), we get the following another result due to Ramanujan (Berndt, 1989) viz.

$$
\sqrt{1-x^{2}}{ }_{2} F_{1}\left[\begin{array}{lll}
\frac{1}{2}, & \frac{1}{2} ; & \\
1 ; & \frac{1}{2}+\frac{x}{1+x^{2}}
\end{array}\right]=\mu_{2} F_{1}\left[\begin{array}{lll}
\frac{1}{2}, & \frac{1}{2} ; \\
\frac{3}{4} ; & & \frac{x^{4}}{x^{4}-1}
\end{array}\right]+2 \eta x_{2} F_{1}\left[\begin{array}{lll}
\frac{1}{2}, & \frac{1}{2} ; \\
\frac{5}{4} ; & \frac{x^{4}}{x^{4}-1}
\end{array}\right] .
$$

Remark 1 For Ramanujan results (9) and (10) see a note by Rakha et al. (2013).

It is interesting to mention here that, in 2001, Rathie and Kim (2001) have obtained two very interesting results closely related to the result (8) due to Gauss. Here we will mention one of their results which we will be using in our present investigation:

$$
\begin{aligned}
& { }_{2} F_{1}\left[\begin{array}{ccc}
r, & m ; & \\
2 m-1 ; & & \frac{4 x}{(1+x)^{2}}
\end{array}\right] \\
& =(1+x)^{2 r}\left\{{ }_{2} F_{1}\left[\begin{array}{cc}
r, & r-m+\frac{3}{2} ; \\
m-\frac{1}{2} ; & x^{2}
\end{array}\right]+\frac{2 r x}{2 m-1}{ }_{2} F_{1}\left[\begin{array}{cc}
r+1, & r-m+\frac{3}{2} ; \\
m+\frac{1}{2} ; & x^{2}
\end{array}\right]\right\} \text {. }
\end{aligned}
$$

Very recently, in a research paper (Krattenthaler et al., 2003), Krattenthaler and Rao developed a technique from which, by using beta integral method, new hypergeometric identities involving one or two variables can be obtained by employing known hypergeometric identities. Using that method, by employing Kummer's formula (5), (by first changing $x$ to $-x$ ) Krattenthaler and Roa (2003) have obtained the following interesting identity viz.

$$
\begin{aligned}
& { }_{3} F_{2}\left[\begin{array}{cccc}
a, & b, & e-d ; & \frac{1}{2} \\
\frac{1}{2}(a+b+1), & e ; &
\end{array}\right] \\
& =\frac{\Gamma\left(\frac{1}{2}\right) \Gamma\left(\frac{1}{2} a+\frac{1}{2} b+\frac{1}{2}\right)}{\Gamma\left(\frac{1}{2} a+\frac{1}{2}\right) \Gamma\left(\frac{1}{2} b+\frac{1}{2}\right)}{ }_{4} F_{3}\left[\begin{array}{ccccc}
\frac{1}{2} a, & \frac{1}{2} b, & \frac{1}{2}+\frac{1}{2} d & \frac{1}{2} d ; & 1 \\
\frac{1}{2}, & \frac{1}{2}+\frac{1}{2} e, & \frac{1}{2} e ; &
\end{array}\right] \\
& -\frac{2 d \Gamma\left(\frac{1}{2}\right) \Gamma\left(\frac{1}{2} a+\frac{1}{2} b+\frac{1}{2}\right)}{e \Gamma\left(\frac{1}{2} a\right) \Gamma\left(\frac{1}{2} b\right)}{ }_{4} F_{3}\left[\begin{array}{ccccc}
\frac{1}{2} a+\frac{1}{2}, & \frac{1}{2} b+\frac{1}{2}, & \frac{1}{2}+\frac{1}{2} d & 1+\frac{1}{2} d ; & \\
2 & \frac{3}{2}, & \frac{1}{2}+\frac{1}{2} e, & \frac{1}{2} e+1 ; &
\end{array}\right] .
\end{aligned}
$$

Further, in (12), if we take $a=b=\frac{1}{2}$, we get

$$
\begin{aligned}
& { }_{3} F_{2}\left[\begin{array}{llll}
\frac{1}{2}, & \frac{1}{2}, & e-d ; \\
1, & e ; & \frac{1}{2}
\end{array}\right] \\
& =\mu_{4} F_{3}\left[\begin{array}{cccc}
\frac{1}{4}, & \frac{1}{4}, & \frac{1}{2} d & \frac{1}{2} d+\frac{1}{2} ; \\
\frac{1}{2}, & \frac{1}{2} e, & \frac{1}{2} e+\frac{1}{2} ; & 1
\end{array}\right]-\eta \frac{d}{e}{ }_{4} F_{3}\left[\begin{array}{rrrr}
\frac{3}{4}, & \frac{3}{4}, & \frac{1}{2} d+\frac{1}{2} & \frac{1}{2} d+1 ; \\
\frac{3}{2}, & \frac{1}{2} e+\frac{1}{2}, & \frac{1}{2} e+1 ; & 1
\end{array}\right]
\end{aligned}
$$


which can also be obtained directly from (6) by employing beta integral method.

On the other hand, in 1996, Lavoie et al. (1996) have generalized the Gauss's second summation theorem (3) and obtained explicit expressions of

$$
{ }_{2} F_{1}\left[\begin{array}{ccc}
a, & b ; & \\
\frac{1}{2}(a+b+i+1) ; & &
\end{array}\right]
$$

for $i=0, \pm 1, \ldots, \pm 5$.

Very recently, Rakha et al. (2014), extended the result (14) for $i=0, \pm 1, \ldots, \pm 9$, and obtained nineteen results closely related to Gauss's second summation Theorem (3) in the form of a single result which is given here

$$
\begin{aligned}
& { }_{2} F_{1}\left[\begin{array}{cc}
a, & b ; \\
\frac{1}{2}(a+b+i+1) ; & \frac{1}{2}
\end{array}\right] \\
= & \frac{\Gamma\left(\frac{1}{2}\right) \Gamma\left(\frac{1}{2} a+\frac{1}{2} b+\frac{1}{2} i+\frac{1}{2}\right) \Gamma\left(\frac{1}{2} a-\frac{1}{2} b-\frac{1}{2} i+\frac{1}{2}\right)}{\Gamma\left(\frac{1}{2} a-\frac{1}{2} b+\frac{1}{2}+\frac{1}{2}|i|\right)} \\
& \times\left[\frac{A_{i}}{\Gamma\left(\frac{1}{2} a+\frac{1}{2}\right) \Gamma\left(\frac{1}{2} b+\frac{1}{2} i+\frac{1}{2}-\left[\frac{1}{2}(1+i)\right]\right)}+\frac{B_{i}}{\Gamma\left(\frac{1}{2} a\right) \Gamma\left(\frac{1}{2} b+\frac{1}{2} i-\left[\frac{1}{2} i\right]\right)}\right]
\end{aligned}
$$

for $i=0, \pm 1, \ldots, \pm 9$. Here $[x]$ denotes the greatest integer less than or equal to $x$ and $|x|$ its absolute value. The coefficients $A_{i}$ and $B_{i}$ are given in Table 1 .

Remark 2 For generalizations of other classical summation theorems, see Lavoie et al. (1992, 1994).

The remainder of the paper will be organized as follows. In Section 2, we shall give the generalization of the Kummer and Ramanujan result in the form

$$
{ }_{2} F_{1}\left[\begin{array}{ccc}
a, & b ; & \\
\frac{1}{2}(a+b+i+1) ; & &
\end{array}\right]
$$

for $i=0, \pm 1, \ldots, \pm 9$.

As special cases, we mentions, five results including the result (5). In Section 3, we shall mention four results closely related to Ramanujan's result (6). In Section 4, we provide four interesting results closely related to the result (8) due to Ramanujan and in Section 5, we shall mention 4 results closely related to Ramanujan's result (9).

In Section 6, we shall mention the natural generalization of the Krattenthaler and Rao's result (12) (and also of (13)) in the form

$$
{ }_{3} F_{2}\left[\begin{array}{cccc}
a, & b, & e-d ; & \\
\frac{1}{2}(a+b+i+1), & e ; & & \frac{1}{2}(1+x)
\end{array}\right]
$$

for $i=0, \pm 1, \ldots, \pm 9$. A few very interesting known as well as new results have also been given.

The results established in this paper are simple, interesting, easily established and may be useful.

We conclude this section with the remain that the results presented in this paper have been verified numerically through the computer algebra system MATHEMATICA, a general system of doing mathematics by computer. 
Table 1. Table for the coefficients $\mathbf{A}_{i}$ and $\mathbf{B}_{i}, i=0 \pm 1, \cdots \pm 9$

\begin{tabular}{|c|c|c|}
\hline i & $\mathbf{A}_{i}$ & $\mathbf{B}_{i}$ \\
\hline 9 & $\begin{array}{l}-16 b^{4}+36 b^{3}(b-a+10)-27 b^{2}(b-a+10)^{2}+ \\
\frac{15}{2} b(b-a+10)^{3}+\frac{23}{16}(b-a+10)^{4}-328 b^{3}+486 b^{2}(b- \\
a+10)-198 b(b-a+10)^{2}+\frac{75}{4}(b-a+10)^{3}- \\
2240 b^{2}+1806 b(b-a+10)-\frac{999}{4}(b-a+10)^{2}- \\
5696 b+1581(b-a+10)-3984\end{array}$ & $\begin{array}{l}16 b^{4}-28 b^{3}(b-a+10)+15 b^{2}(b-a+10)^{2}-\frac{5}{2} b(b- \\
a+10)^{3}+\frac{1}{16}(b-a+10)^{4}+248 b^{3}-258 b^{2}(b-a+10)+ \\
60 b(b-a+10)^{2}-\frac{5}{4}(b-a+10)^{3}+1160 b^{2}-514 b(b- \\
a+10)+\frac{35}{4}(b-a+10)^{2}+1576 b-25(b-a+10)+24\end{array}$ \\
\hline 8 & $\begin{array}{l}8 b^{4}-16 b^{3}(b-a+9)+10 b^{2}(b-a+9)^{2}-2 b(b- \\
a+9)^{3}+\frac{1}{16}(b-a+9)^{4}+128 b^{3}-156 b^{2}(b-a+9)+ \\
44 b(b-a+9)^{2}-\frac{5}{4}(b-a+9)^{3}+624 b^{2}-336 b(b- \\
a+9)+\frac{35}{4}(b-a+9)^{2}+896 b-25(b-a+9)+24\end{array}$ & $\begin{array}{l}(b-a+9)^{3}-10 b(b-a+9)^{2}+24 b^{2}(b-a+9)- \\
16 b^{3}-192 b^{2}+156 b(b-a+9)-22(b-a+9)^{2}- \\
640 b+176(b-a+9)-512\end{array}$ \\
\hline 7 & $\begin{array}{l}\frac{7}{8}(b-a+8)^{3}-7 b(b-a+8)^{2}+14 b^{2}(b-a+8)- \\
8 b^{3}-100 b^{2}+98 b(b-a+8)-\frac{35}{2}(b-a+8)^{2}- \\
352 b+\frac{245}{2}(b-a+8)-302\end{array}$ & $\begin{array}{l}8 b^{3}-10 b^{2}(b-a+8)+3 b(b-a+8)^{2}-\frac{1}{8}(b-a+ \\
8)^{3}+68 b^{2}-38 b(b-a+8)+\frac{3}{2}(b-a+8)^{2}+128 b- \\
\frac{11}{2}(b-a+8)+6\end{array}$ \\
\hline 6 & $\begin{array}{l}4 b^{3}-6 b^{2}(b-a+7)+\frac{9}{4} b(b-a+7)^{2}-\frac{1}{8}(b-a+ \\
7)^{3}+36 b^{2}-\frac{51}{2} b(b-a+7)+\frac{3}{2}(b-a+7)^{2}+74 b- \\
\frac{11}{2}(b-a+7)+6\end{array}$ & $25 b-17 a-18 a b-\frac{3}{2}(b-a+7)^{2}+67$ \\
\hline 5 & $\frac{1}{4}\left(-8+10 a-5 a^{2}+6 b-10 a b-b^{2}\right)$ & $\frac{1}{4}\left(8-6 a+a^{2}-10 b+10 a b+5 b^{2}\right)$ \\
\hline 4 & $\frac{1}{4}\left(3-4 a+a^{2}-4 b+6 a b+b^{2}\right)$ & $2(1-a-b)$ \\
\hline 3 & $\frac{1}{2}(2-3 a-b)$ & $\frac{1}{2}(a+3 b-2)$ \\
\hline 2 & $\frac{1}{2}(a+b-1)$ & -2 \\
\hline 1 & -1 & 1 \\
\hline 0 & 1 & 0 \\
\hline-1 & 1 & 1 \\
\hline-2 & $\frac{1}{2}(a+b-1)$ & 2 \\
\hline-3 & $\frac{1}{2}(3 a+b-2)$ & $\frac{1}{2}(a+3 b-2)$ \\
\hline-4 & $\frac{1}{4}\left(3-4 a+a^{2}-4 b+6 a b+b^{2}\right)$ & $2(a+b-1)$ \\
\hline-5 & $\frac{1}{4}\left(8-10 a+5 a^{2}-6 b+10 a b+b^{2}\right)$ & $\frac{1}{4}\left(8-6 a+a^{2}-10 b+10 a b+5 b^{2}\right)$ \\
\hline-6 & $\begin{array}{l}4 b^{3}-6 b^{2}(b-a-5)+\frac{9}{4} b(b-a-5)^{2}-\frac{1}{8}(b-a- \\
5)^{3}-36 b^{2}+\frac{57}{2} b(b-a-5)-3(b-a-5)^{2}+92 b- \\
\frac{47}{2}(b-a-5)-60\end{array}$ & $8 a b+11 b-19 a-31+\frac{3}{2}(b-a-5)^{2}$ \\
\hline-7 & $\begin{array}{l}8 b^{3}-14 b^{2}(b-a-6)+7 b(b-a-6)^{2}-\frac{7}{8}(b-a- \\
6)^{3}-96 b^{2}+98 b(b-a-6)-\frac{77}{4}(b-a-6)^{2}+352 b- \\
147(b-a-6)-384\end{array}$ & $\begin{array}{l}8 b^{3}-10 b^{2}(b-a-6)+3 b(b-a-6)^{2}-\frac{1}{8}(b-a- \\
6)^{3}-72 b^{2}+46 b(b-a-6)-\frac{15}{4}(b-a-6)^{2}+184 b- \\
37(b-a-6)-120\end{array}$ \\
\hline-8 & $\begin{array}{l}8 b^{4}-16 b^{3}(b-a-7)+10 b^{2}(b-a-7)^{2}-2 b(b- \\
a-7)^{3}+\frac{1}{16}(b-a-7)^{4}-128 b^{3}+164 b^{2}(b-a-7)- \\
52 b(b-a-7)^{2}+\frac{11}{4}(b-a-7)^{3}+688 b^{2}-464 b(b- \\
a-7)+\frac{179}{4}(b-a-7)^{2}-1408 b+319(b-a-7)+840\end{array}$ & $\begin{array}{l}16 b^{3}-24 b^{2}(b-a-7)+10 b(b-a-7)^{2}-(b-a- \\
7)^{3}-192 b^{2}+164 b(b-a-7)-26(b-a-7)^{2}+ \\
704 b-240(b-a-7)-768\end{array}$ \\
\hline-9 & $\begin{array}{l}16 b^{4}-36 b^{3}(b-a-8)+27 b^{2}(b-a-8)^{2}-\frac{15}{2} b(b- \\
a-8)^{3}+\frac{9}{16}(b-a-8)^{4}-320 b^{3}+486 b^{2}(b-a- \\
8)-207 b(b-a-8)^{2}+\frac{87}{4}(b-a-8)^{3}+2240 b^{2}- \\
1968 b(b-a-8)+\frac{1323}{4}(b-a-8)^{2}-6400 b+2307(b- \\
a-8)+6144\end{array}$ & $\begin{array}{l}16 b^{4}-28 b^{3}(b-a-8)+15 b^{2}(b-a-8)^{2}-\frac{5}{2} b(b- \\
a-8)^{3}+\frac{1}{16}(b-a-8)^{4}-256 b^{3}+282 b^{2}(b-a-8)- \\
75 b(b-a-8)^{2}+\frac{13}{4}(b-a-8)^{3}+1376 b^{2}-784 b(b- \\
a-8)+\frac{251}{4}(b-a-8)^{2}-2816 b+533(b-a-8)+1680\end{array}$ \\
\hline
\end{tabular}

\section{Generalization of Kummer and Ramanujan Result (5)}

The generalization of Kummer and Ramanujan result (5) containing 19 results in the form of a single result to be 
established is given by

$$
\begin{aligned}
{ }_{2} F_{1}\left[\begin{array}{c}
a, \quad b ; \\
\frac{1}{2}(a+b+i+1) ;
\end{array}\right] & \frac{\Gamma\left(\frac{1}{2}\right) \Gamma\left(\frac{1}{2} a+\frac{1}{2} b+\frac{1}{2} i+\frac{1}{2}\right) \Gamma\left(\frac{1}{2} a-\frac{1}{2} b-\frac{1}{2} i+\frac{1}{2}\right)}{\Gamma\left(\frac{1}{2} a-\frac{1}{2} b+\frac{1}{2}+\frac{1}{2}|i|\right)} \\
& \times\left\{\sum_{j=0}^{\infty} \frac{\left(\frac{1}{2} a\right)_{j}\left(\frac{1}{2} a+\frac{1}{2}\right)_{j}\left(\frac{1}{2} b\right)_{j}\left(\frac{1}{2} b+\frac{1}{2}\right)_{j} x^{2 j}}{\left(\frac{1}{2}\right)_{j} j !}\right. \\
& \cdot\left[\frac{C_{i}}{\Gamma\left(\frac{1}{2} a+\frac{1}{2}\right) \Gamma\left(\frac{1}{2} b+\frac{1}{2} i+\frac{1}{2}-\left[\frac{i+1}{2}\right]\right)\left(\frac{1}{2} a+\frac{1}{2}\right)_{j}\left(\frac{1}{2} b+\frac{1}{2} i+\frac{1}{2}-\left[\frac{i+1}{2}\right]\right)_{j}}\right. \\
& \left.+\frac{D_{i}}{\Gamma\left(\frac{1}{2} a\right) \Gamma\left(\frac{1}{2} b+\frac{1}{2} i-\left[\frac{i}{2}\right]\right)\left(\frac{1}{2} a\right)_{j}\left(\frac{1}{2} b+\frac{1}{2} i-\left[\frac{i}{2}\right]\right)_{j}}\right] \\
& +\frac{a b x}{2} \sum_{j=0}^{\infty} \frac{\left(\frac{1}{2} a+\frac{1}{2}\right)_{j}\left(\frac{1}{2} a+1\right)_{j}\left(\frac{1}{2} b+\frac{1}{2}\right)_{j}\left(\frac{1}{2} b+1\right)_{j}}{j !} x^{2 j} \\
& \cdot\left[\frac{E_{i}}{\Gamma\left(\frac{1}{2} a+1\right) \Gamma\left(\frac{1}{2} b+1+\frac{1}{2} i-\left[\frac{i+1}{2}\right]\right)\left(\frac{1}{2} a+1\right)_{j}\left(\frac{1}{2} b+1+\frac{1}{2} i-\left[\frac{i+1}{2}\right]\right)_{j}}\right. \\
& +\frac{F_{i}}{\left.\Gamma\left(\frac{1}{2} a+\frac{1}{2}\right) \Gamma\left(\frac{1}{2} b+\frac{1}{2}+\frac{1}{2} i-\left[\frac{i}{2}\right]\right)\left(\frac{1}{2} a+\frac{1}{2}\right)_{j}\left(\frac{1}{2} b+\frac{1}{2}+\frac{1}{2} i-\left[\frac{i}{2}\right]\right)_{j}\right]}
\end{aligned}
$$

for $i=0, \pm 1, \ldots, \pm 9$.

The coefficients $C_{i}$ and $D_{i}$ can be obtained from the Table 1 by changing $a$ to $a+2 j$ and $b$ to $b+2 j$ and the coefficients $E_{i}$ and $F_{i}$ can be obtained from the Table 1 by changing $a$ to $a+2 j+1$ and $b$ to $b+2 j+1$ respectively.

Proof. In order to derive the result (16), we proceed as follows. Denoting the left-hand side of (16) by $S$, expressing ${ }_{2} F_{1}$ as an infinite series with the help of its definition, we have

$$
S=\sum_{k=0}^{\infty} \frac{(a)_{k}(b)_{k}}{\left(\frac{1}{2}(a+b+i+1)\right)_{k} 2^{k} k !}(1+x)^{k}
$$

using Binomial theorem and after some simplification

$$
S=\sum_{k=0}^{\infty} \sum_{j=0}^{k} \frac{(a)_{k}(b)_{k}}{\left(\frac{1}{2}(a+b+i+1)\right)_{k} 2^{k}} \frac{x^{j}}{j !(k-j) !} .
$$

Now, if we use the known result (Srivastava et al., 1985)

$$
\sum_{k=0}^{\infty} \sum_{j=0}^{k} A_{j, k} \frac{x^{j}}{j !} \frac{y^{k}}{(x-j) !}=\sum_{j=0}^{\infty} \frac{x^{j}}{j !} \sum_{k=j}^{\infty} A_{j, k} \frac{y^{k}}{(x-j) !}
$$

we get after some little algebra

$$
S=\sum_{j=0}^{\infty} \frac{x^{j}}{j !} \sum_{k=j}^{\infty} \frac{(a)_{k}(b)_{k}}{\left(\frac{1}{2}(a+b+i+1)\right)_{k} 2^{k}(k-j) !} .
$$

Now, changing $k$ to $j+k$ and using the well known identity

$$
(a)_{j+k}=(a)_{j}(a+j)_{k}
$$

we get after some simplification

$$
S=\sum_{j=0}^{\infty} \frac{(a)_{j}(b)_{j}}{\left(\frac{1}{2}(a+b+i+1)\right)_{k} 2^{j}} \frac{x^{j}}{j !} \sum_{k=j}^{\infty} \frac{(a+j)_{k}(b+j)_{k}}{\left(\frac{1}{2}(a+b+i+1)+j\right)_{k} 2^{k} k !} .
$$


Now, summing up the inner series with the help of the definition of ${ }_{2} F_{1}$, we have

$$
S=\sum_{j=0}^{\infty} \frac{(a)_{j}(b)_{j}}{\left(\frac{1}{2}(a+b+i+1)\right)_{k} 2^{j}} \frac{x^{j}}{j !}{ }_{2} F_{1}\left[\begin{array}{ccc}
a+j, & b+j ; & \\
\frac{1}{2}(a+b+i+1+2 j) ; & & \frac{1}{2}
\end{array}\right] .
$$

Now, separating the series into even and odd powers of $x$ and then using the result (15) and using the identity

$$
(a)_{2 n}=2^{2 n}\left(\frac{1}{2} a\right)_{n}\left(\frac{1}{2} a+\frac{1}{2}\right)_{n}, \quad\left(n \in \mathbb{N}_{0}\right)
$$

we get, after much simplification, the right-hand side of (16). This completes the proof of (16).

\section{Special Cases}

Here we shall mention some of the interesting special cases of our main result (16). It is easy to see that, if in (16), we take $i=0, \pm 1, \pm 2$, we get, after some simplification, the following interesting results:

1) For $i=0$

$$
\begin{aligned}
& { }_{2} F_{1}\left[\begin{array}{crr}
a, & b ; & \\
\frac{1}{2}(a+b+1) ; & & \frac{1+x}{2}
\end{array}\right]=\frac{\Gamma\left(\frac{1}{2}\right) \Gamma\left(\frac{1}{2} a+\frac{1}{2} b+\frac{1}{2}\right)}{\Gamma\left(\frac{1}{2} a+\frac{1}{2}\right) \Gamma\left(\frac{1}{2} b+\frac{1}{2}\right)}{ }_{2} F_{1}\left[\begin{array}{ccc}
\frac{1}{2} a, & \frac{1}{2} b ; & \\
\frac{1}{2} ; & & x^{2}
\end{array}\right] \\
& +\frac{2 x \Gamma\left(\frac{1}{2}\right) \Gamma\left(\frac{1}{2} a+\frac{1}{2} b+\frac{1}{2}\right)}{\Gamma\left(\frac{1}{2} a\right) \Gamma\left(\frac{1}{2} b\right)}{ }_{2} F_{1}\left[\begin{array}{ccc}
\frac{1}{2} a+\frac{1}{2}, & \frac{1}{2} b+\frac{1}{2} ; \\
\frac{3}{2} ; & x^{2}
\end{array}\right] .
\end{aligned}
$$

for $x \in U$.

2) For $i=1$

$$
\begin{aligned}
& { }_{2} F_{1}\left[\begin{array}{crr}
a, & b ; & \\
\frac{1}{2}(a+b+2) ; & & \frac{1+x}{2}
\end{array}\right]=\frac{2}{(a-b)} \Gamma\left(\frac{1}{2}\right) \Gamma\left(\frac{1}{2} a+\frac{1}{2} b+1\right) \\
& \times\left[\frac{1}{\Gamma\left(\frac{1}{2} a\right) \Gamma\left(\frac{1}{2} b+\frac{1}{2}\right)}{ }_{2} F_{1}\left[\begin{array}{ccc}
\frac{1}{2} a+\frac{1}{2}, & \frac{1}{2} b ; \\
\frac{1}{2} ; &
\end{array}\right]\right. \\
& -\frac{1}{\Gamma\left(\frac{1}{2} a+\frac{1}{2}\right) \Gamma\left(\frac{1}{2} b\right)}{ }_{2} F_{1}\left[\begin{array}{lll}
\frac{1}{2} a, & \frac{1}{2} b ; & \\
\frac{1}{2} ; & & x^{2}
\end{array}\right] \\
& -\frac{a b x}{2 \Gamma\left(\frac{1}{2} a+1\right) \Gamma\left(\frac{1}{2} b+\frac{1}{2}\right)}{ }_{2} F_{1}\left[\begin{array}{ccc}
\frac{1}{2} a+\frac{1}{2}, & \frac{1}{2} b+1 ; \\
\frac{3}{2} ; & x^{2}
\end{array}\right] \\
& +\frac{a b x}{2 \Gamma\left(\frac{1}{2} a+\frac{1}{2}\right) \Gamma\left(\frac{1}{2} b+1\right)}{ }_{2} F_{1}\left[\begin{array}{ccc}
\frac{1}{2} a+1, & \frac{1}{2} b+\frac{1}{2} ; & x^{2} \\
\frac{3}{2} ; &
\end{array}\right] \text {. }
\end{aligned}
$$

for $x \in U$.

3) For $i=-1$

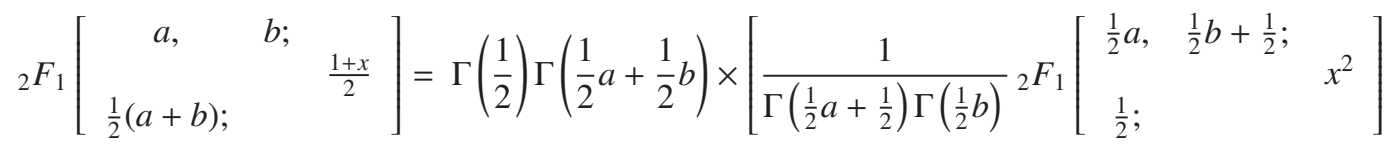

$$
\begin{aligned}
& +\frac{1}{\Gamma\left(\frac{1}{2} a\right) \Gamma\left(\frac{1}{2} b+\frac{1}{2}\right)}{ }_{2} F_{1}\left[\begin{array}{cc}
\frac{1}{2} a+\frac{1}{2}, & \frac{1}{2} b ; \\
\frac{1}{2} ; & x^{2}
\end{array}\right]
\end{aligned}
$$




$$
\begin{aligned}
& +\frac{a b x}{2 \Gamma\left(\frac{1}{2} a+1\right) \Gamma\left(\frac{1}{2} b+\frac{1}{2}\right)}{ }_{2} F_{1}\left[\begin{array}{ccc}
\frac{1}{2} a+\frac{1}{2}, & \frac{1}{2} b+1 ; & \\
\frac{3}{2} ; & & x^{2}
\end{array}\right] \\
& \left.+\frac{a b x}{2 \Gamma\left(\frac{1}{2} a+1\right) \Gamma\left(\frac{1}{2} b+\frac{1}{2}\right)}{ }_{2} F_{1}\left[\begin{array}{ccc}
\frac{1}{2} a+1, & \frac{1}{2} b+\frac{1}{2} ; & \\
\frac{3}{2} ; & & x^{2}
\end{array}\right]\right] .
\end{aligned}
$$

for $x \in U$.

4) For $i=2$

$$
\begin{aligned}
& { }_{2} F_{1}\left[\begin{array}{ccc}
a, & b ; & \\
\frac{1}{2}(a+b+3) ; & & \frac{1+x}{2}
\end{array}\right]=\frac{\Gamma\left(\frac{1}{2}\right) \Gamma\left(\frac{1}{2} a+\frac{1}{2} b+\frac{3}{2}\right) \Gamma\left(\frac{1}{2} a-\frac{1}{2} b-\frac{1}{2}\right)}{\Gamma\left(\frac{1}{2} a-\frac{1}{2} b+\frac{3}{2}\right)} \\
& \times\left[\frac{(b+a-1)}{2 \Gamma\left(\frac{1}{2} a+\frac{1}{2}\right) \Gamma\left(\frac{1}{2} b+\frac{1}{2}\right)}{ }_{2} F_{1}\left[\begin{array}{lll}
\frac{1}{2} a, & \frac{1}{2} b ; \\
\frac{1}{2} ; & x^{2}
\end{array}\right]\right. \\
& -\frac{2}{\Gamma\left(\frac{1}{2} a\right) \Gamma\left(\frac{1}{2} b\right)}{ }_{2} F_{1}\left[\begin{array}{ccc}
\frac{1}{2} a+\frac{1}{2}, & \frac{1}{2} b+\frac{1}{2} ; \\
\frac{1}{2} ; & x^{2}
\end{array}\right] \\
& +\frac{a b(a+b+1) x}{4 \Gamma\left(\frac{1}{2} a+1\right) \Gamma\left(\frac{1}{2} b+1\right)}{ }_{2} F_{1}\left[\begin{array}{ccc}
\frac{1}{2} a+\frac{1}{2}, & \frac{1}{2} b+\frac{1}{2} ; \\
\frac{3}{2} ; & x^{2}
\end{array}\right] \\
& -\frac{a b x}{\Gamma\left(\frac{1}{2} a+\frac{1}{2}\right) \Gamma\left(\frac{1}{2} b+\frac{1}{2}\right)}{ }_{2} F_{1}\left[\begin{array}{ccc}
\frac{1}{2} a+1, & \frac{1}{2} b+1 ; & x^{2} \\
\frac{3}{2} ; &
\end{array}\right] \\
& +\frac{a b x^{2}}{\Gamma\left(\frac{1}{2} a+\frac{1}{2}\right) \Gamma\left(\frac{1}{2} b+\frac{1}{2}\right)}{ }_{2} F_{1}\left[\begin{array}{ccc}
\frac{1}{2} a+1, & \frac{1}{2} b+1 ; \\
\frac{3}{2} ; & x^{2}
\end{array}\right] \\
& +\frac{a b(a+1)(b+1) x^{3}}{6 \Gamma\left(\frac{1}{2} a+1\right) \Gamma\left(\frac{1}{2} b+1\right)}{ }_{2} F_{1}\left[\begin{array}{ccc}
\frac{1}{2} a+\frac{3}{2}, & \frac{1}{2} b+\frac{3}{2} ; \\
\frac{5}{2} ; & & x^{2}
\end{array}\right] \text {. }
\end{aligned}
$$

for $x \in U$.

5) For $i=-2$

$$
\begin{aligned}
& { }_{2} F_{1}\left[\begin{array}{crr}
a, & b ; & \\
\frac{1}{2}(a+b-1) ; & & \frac{1+x}{2}
\end{array}\right]=\Gamma\left(\frac{1}{2}\right) \Gamma\left(\frac{1}{2} a+\frac{1}{2} b-\frac{1}{2}\right) \\
& \times\left[\frac{(a+b-1)}{2 \Gamma\left(\frac{1}{2} a+\frac{1}{2}\right) \Gamma\left(\frac{1}{2} b+\frac{1}{2}\right)}{ }_{2} F_{1}\left[\begin{array}{lll}
\frac{1}{2} a, & \frac{1}{2} b ; & \\
\frac{1}{2} ; & & x^{2}
\end{array}\right]\right. \\
& +\frac{2}{\Gamma\left(\frac{1}{2} a\right) \Gamma\left(\frac{1}{2} b\right)}{ }_{2} F_{1}\left[\begin{array}{ccc}
\frac{1}{2} a+\frac{1}{2}, & \frac{1}{2} b+\frac{1}{2} ; & x^{2} \\
\frac{1}{2} ; &
\end{array}\right] \\
& +\frac{a b x}{\Gamma\left(\frac{1}{2} a+\frac{1}{2}\right) \Gamma\left(\frac{1}{2} b+\frac{1}{2}\right)}{ }_{2} F_{1}\left[\begin{array}{ccc}
\frac{1}{2} a+1, & \frac{1}{2} b+1 ; \\
\frac{3}{2} ; & x^{2}
\end{array}\right] \\
& +\frac{a b(a+b+1) x}{4 \Gamma\left(\frac{1}{2} a+1\right) \Gamma\left(\frac{1}{2} b+1\right)}{ }_{2} F_{1}\left[\begin{array}{ccc}
\frac{1}{2} a+\frac{1}{2}, & \frac{1}{2} b+\frac{1}{2} ; \\
\frac{3}{2} ; & & x^{2}
\end{array}\right]
\end{aligned}
$$




$$
\begin{aligned}
& +\frac{a b x^{2}}{\Gamma\left(\frac{1}{2} a+\frac{1}{2}\right) \Gamma\left(\frac{1}{2} b+\frac{1}{2}\right)}{ }_{2} F_{1}\left[\begin{array}{ccc}
\frac{1}{2} a+1, & \frac{1}{2} b+1 ; & \\
\frac{3}{2} ; & & x^{2}
\end{array}\right] \\
& \left.+\frac{a b(a+1)(b+1) x^{3}}{6 \Gamma\left(\frac{1}{2} a+1\right) \Gamma\left(\frac{1}{2} b+1\right)}{ }_{2} F_{1}\left[\begin{array}{ccc}
\frac{1}{2} a+\frac{3}{2}, & \frac{1}{2} b+\frac{3}{2} ; & \\
\frac{5}{2} ; & & x^{2}
\end{array}\right]\right] .
\end{aligned}
$$

6) In (17), if we take $a=b=\frac{1}{2}$, we get after some simplification, the Ramanujan's result (6).

7) In (18), if we take $a=\frac{1}{2}, b=\frac{3}{2}$, we get

$$
\begin{aligned}
{ }_{2} F_{1}\left[\begin{array}{ccc}
\frac{1}{2}, & \frac{3}{2} ; \\
2 ; & \frac{1+x}{2}
\end{array}\right]= & 2 \mu_{2} F_{1}\left[\begin{array}{lll}
\frac{1}{4}, & \frac{5}{4} ; \\
\frac{1}{2} ; & & x^{2}
\end{array}\right]-4 \eta_{2} F_{1}\left[\begin{array}{ccc}
\frac{3}{4}, & \frac{3}{4} ; & \\
\frac{1}{2} ; & x^{2}
\end{array}\right] \\
& -\mu x_{2} F_{1}\left[\begin{array}{ccc}
\frac{5}{4}, & \frac{5}{4} ; \\
\frac{3}{2} ; & & x^{2}
\end{array}\right]+6 \eta x_{2} F_{1}\left[\begin{array}{ccc}
\frac{3}{4}, & \frac{7}{4} ; & x^{2} \\
\frac{3}{2} ; &
\end{array}\right] .
\end{aligned}
$$

8) In (19), if we take $a=\frac{1}{2}, b=\frac{3}{2}$, we get

$$
\begin{aligned}
{ }_{2} F_{1}\left[\begin{array}{rr}
\frac{1}{2}, & \frac{3}{2} ; \\
1 ; & \frac{1+x}{2}
\end{array}\right]= & \mu_{2} F_{1}\left[\begin{array}{lll}
\frac{1}{4}, & \frac{5}{4} ; \\
\frac{1}{2} ; & x^{2}
\end{array}\right]+2 \eta_{2} F_{1}\left[\begin{array}{ll}
\frac{3}{4}, & \frac{3}{4} ; \\
\frac{1}{2} ; & x^{2}
\end{array}\right] \\
& +\frac{1}{2} \mu x_{2} F_{1}\left[\begin{array}{ll}
\frac{5}{4}, & \frac{5}{4} ; \\
\frac{3}{2} ; & x^{2}
\end{array}\right]+3 \eta x_{2} F_{1}\left[\begin{array}{lll}
\frac{3}{4}, & \frac{7}{4} ; \\
\frac{3}{2} ; & x^{2}
\end{array}\right] .
\end{aligned}
$$

9) In (20), if we take $a=\frac{1}{2}, b=\frac{5}{2}$, we get

$$
\begin{aligned}
& { }_{2} F_{1}\left[\begin{array}{lll}
\frac{1}{2}, & \frac{5}{2} ; & \\
3 ; & \frac{1+x}{2}
\end{array}\right]=\frac{32}{9} \mu_{2} F_{1}\left[\begin{array}{ll}
\frac{1}{4}, & \frac{5}{4} ; \\
\frac{1}{2} ; &
\end{array}\right]-\frac{32}{3} \eta_{2} F_{1}\left[\begin{array}{lll}
\frac{3}{4}, & \frac{7}{4} ; \\
\frac{1}{2} ; & & x^{2}
\end{array}\right] \\
& -\frac{40}{9} \mu x_{2} F_{1}\left[\begin{array}{ll}
\frac{5}{4}, & \frac{9}{4} ; \\
\frac{3}{2} ; & x^{2}
\end{array}\right]+\frac{64}{3} \eta x_{2} F_{1}\left[\begin{array}{ll}
\frac{3}{4}, & \frac{7}{4} ; \\
\frac{3}{2} ; & x^{2}
\end{array}\right] \\
& +\frac{40}{9} \mu x_{2}^{2} F_{1}\left[\begin{array}{ll}
\frac{5}{4}, & \frac{9}{4} ; \\
\frac{3}{2} ; & x^{2}
\end{array}\right]+\frac{56}{3} \eta x^{3}{ }_{2} F_{1}\left[\begin{array}{ccc}
\frac{7}{4}, & \frac{11}{4} ; \\
\frac{5}{2} ; & & x^{2}
\end{array}\right] .
\end{aligned}
$$

10) In (21), if we take $a=\frac{1}{2}, b=\frac{5}{2}$, we get

$$
\begin{aligned}
& { }_{2} F_{1}\left[\begin{array}{lll}
\frac{1}{2}, & \frac{5}{2} ; \\
1 ; & \frac{1+x}{2}
\end{array}\right]=\frac{4}{3} \mu_{2} F_{1}\left[\begin{array}{lll}
\frac{1}{4}, & \frac{5}{4} ; \\
\frac{1}{2} ; & & x^{2}
\end{array}\right]+4 \eta_{2} F_{1}\left[\begin{array}{lll}
\frac{3}{4}, & \frac{7}{4} ; & \\
\frac{1}{2} ; & & x^{2}
\end{array}\right] \\
& +\frac{5}{3} \mu x_{2} F_{1}\left[\begin{array}{ll}
\frac{5}{4}, & \frac{9}{4} ; \\
\frac{3}{2} ; & x^{2}
\end{array}\right]+8 \eta x_{2} F_{1}\left[\begin{array}{lll}
\frac{3}{4}, & \frac{7}{4} ; \\
\frac{3}{2} ; & & x^{2}
\end{array}\right] \\
& +\frac{5}{3} \mu x_{2}^{2} F_{1}\left[\begin{array}{cc}
\frac{5}{4}, & \frac{9}{4} ; \\
\frac{3}{2} ; & x^{2}
\end{array}\right]+7 \eta x_{2}^{3} F_{1}\left[\begin{array}{lll}
\frac{7}{4}, & \frac{11}{4} ; \\
\frac{5}{2} ; & & x^{2}
\end{array}\right] .
\end{aligned}
$$

We conclude this section with the remark that the results (22) to (25) are closely related to the Ramanujan's result (6). The results (17) to (19) and (22) and (23) are also recorded in (Choi et al., 2011). 


\section{Results Closely Related to Ramanujan's Results (9)}

In this section, we shall establish the following interesting results closely related to the Ramanujan's result (9). These are

1)

$$
\begin{aligned}
{ }_{2} F_{1}\left[\begin{array}{cc}
\frac{1}{2}, \frac{3}{2} ; & \frac{1}{2}+\frac{x}{1+x^{2}}
\end{array}\right]= & 2 \mu\left(1+x^{2}\right)^{\frac{5}{2}}{ }_{2} F_{1}\left[\begin{array}{ccc}
\frac{3}{2}, & \frac{5}{4} ; & \\
2 ; & & x^{4}
\end{array}\right]-4 \eta\left(1+x^{2}\right)^{\frac{3}{2}}{ }_{2} F_{1}\left[\begin{array}{cc}
\frac{3}{2}, & \frac{3}{4} ; \\
\frac{1}{4} ; & x^{4}
\end{array}\right] \\
& -2 \mu x\left(1+x^{2}\right)^{\frac{3}{2}}{ }_{2} F_{1}\left[\begin{array}{ccc}
\frac{3}{2}, & \frac{5}{4} ; & \\
\frac{3}{4} ; & x^{4}
\end{array}\right]+12 \eta x\left(1+x^{2}\right)^{\frac{5}{2}}{ }_{2} F_{1}\left[\begin{array}{ccc}
\frac{3}{2}, & \frac{7}{4} ; & \\
\frac{5}{4} ; & x^{4}
\end{array}\right] \\
& -12 \eta x^{2}\left(1+x^{2}\right)^{\frac{3}{2}}{ }_{2} F_{1}\left[\begin{array}{ccc}
\frac{3}{2}, & \frac{7}{4} ; & \\
\frac{5}{4} ; & & x^{4}
\end{array}\right]-\frac{10}{3} \mu x^{3}\left(1+x^{2}\right)^{\frac{3}{2}}{ }_{2} F_{1}\left[\begin{array}{lll}
\frac{3}{2}, & \frac{9}{4} ; & \\
\frac{7}{4} ; &
\end{array}\right] .
\end{aligned}
$$

2)

$$
\begin{aligned}
{ }_{2} F_{1}\left[\begin{array}{cc}
\frac{1}{2}, & \frac{3}{2} ; \\
1 ; & \frac{1}{2}+\frac{x}{1+x^{2}}
\end{array}\right]= & \mu\left(1+x^{2}\right)^{\frac{5}{2}}{ }_{2} F_{1}\left[\begin{array}{ccc}
\frac{3}{2}, & \frac{5}{4} ; \\
\frac{3}{4} ; & x^{4}
\end{array}\right]+2 \eta\left(1+x^{2}\right)^{\frac{3}{2}}{ }_{2} F_{1}\left[\begin{array}{cc}
\frac{3}{2}, & \frac{3}{4} ; \\
\frac{1}{4} ; & x^{4}
\end{array}\right] \\
& +6 \eta x\left(1+x^{2}\right)^{\frac{5}{2}}{ }_{2} F_{1}\left[\begin{array}{ccc}
\frac{3}{2}, & \frac{7}{4} ; & \\
\frac{5}{4} ; & x^{4}
\end{array}\right]+\mu x\left(1+x^{2}\right)^{\frac{3}{2}}{ }_{2} F_{1}\left[\begin{array}{ccc}
\frac{3}{2}, & \frac{5}{4} ; & \\
\frac{3}{4} ; & x^{4}
\end{array}\right] \\
& +6 \eta x^{2}\left(1+x^{2}\right)^{\frac{3}{2}}{ }_{2} F_{1}\left[\begin{array}{ccc}
\frac{3}{2}, & \frac{7}{4} ; & \\
\frac{5}{4} ; & & x^{4}
\end{array}\right]+\frac{5}{3} \mu x^{3}\left(1+x^{2}\right)^{\frac{3}{2}}{ }_{2} F_{1}\left[\begin{array}{ccc}
\frac{3}{2}, & \frac{9}{4} ; \\
\frac{7}{4} ; & x^{4}
\end{array}\right] .
\end{aligned}
$$

3)

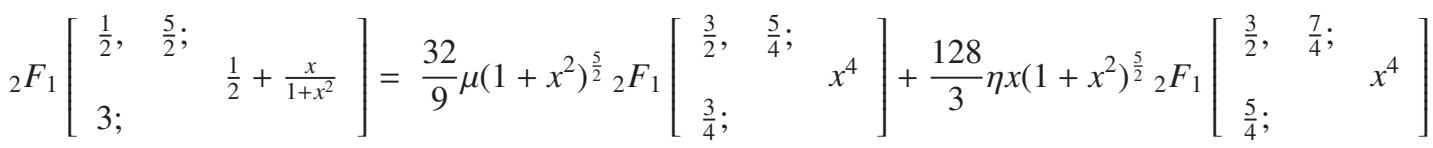

$$
\begin{aligned}
& -\frac{224}{3} \eta x^{2}\left(1+x^{2}\right)^{\frac{7}{2}}{ }_{2} F_{1}\left[\begin{array}{ll}
\frac{5}{2}, & \frac{11}{4} ; \\
\frac{5}{4} ; & x^{4}
\end{array}\right]-\frac{32}{3} \eta\left(1+x^{2}\right)^{\frac{7}{2}}{ }_{2} F_{1}\left[\begin{array}{lll}
\frac{5}{2}, & \frac{7}{4} ; & \\
\frac{1}{4} ; & & x^{4}
\end{array}\right] \\
& -\frac{80}{3} \mu x^{3}\left(1+x^{2}\right)^{\frac{7}{2}}{ }_{2} F_{1}\left[\begin{array}{ll}
\frac{5}{2}, & \frac{13}{4} ; \\
\frac{7}{4} ; & x^{4}
\end{array}\right]-\frac{80}{9} \mu x\left(1+x^{2}\right)^{\frac{7}{2}}{ }_{2} F_{1}\left[\begin{array}{lll}
\frac{5}{2}, & \frac{9}{4} ; & \\
\frac{3}{4} ; &
\end{array}\right] \\
& +\frac{160}{3} \mu x^{4}\left(1+x^{2}\right)^{\frac{5}{2}}{ }_{2} F_{1}\left[\begin{array}{ll}
\frac{5}{2}, & \frac{13}{4} ; \\
\frac{7}{4} ; & x^{4}
\end{array}\right]+\frac{160}{9} \mu x^{2}\left(1+x^{2}\right)^{\frac{5}{2}}{ }_{2} F_{1}\left[\begin{array}{lll}
\frac{5}{2}, & \frac{9}{4} ; & \\
\frac{3}{4} ; &
\end{array}\right]
\end{aligned}
$$

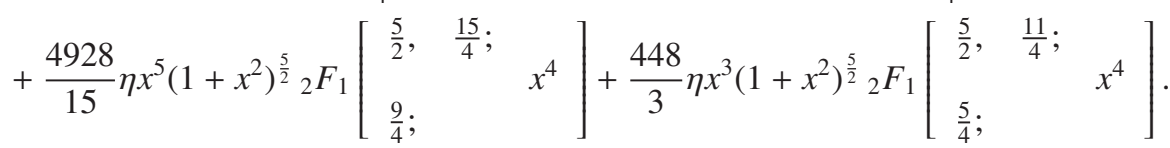

4)

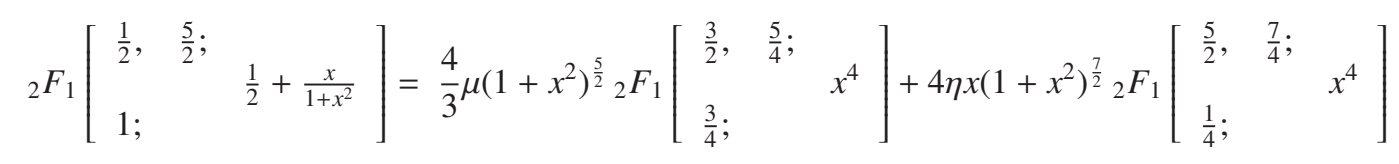




$$
\begin{aligned}
& +\frac{10}{3} \mu x\left(1+x^{2}\right)^{\frac{7}{2}}{ }_{2} F_{1}\left[\begin{array}{ll}
\frac{5}{2}, & \frac{9}{4} ; \\
\frac{3}{4} ; & x^{4}
\end{array}\right]+16 \eta x\left(1+x^{2}\right)^{\frac{5}{2}}{ }_{2} F_{1}\left[\begin{array}{lll}
\frac{3}{2}, & \frac{7}{4} ; & \\
\frac{5}{4} ; & & x^{4}
\end{array}\right] \\
& +\frac{20}{3} \mu x^{2}\left(1+x^{2}\right)^{\frac{5}{2}}{ }_{2} F_{1}\left[\begin{array}{ll}
\frac{5}{2}, & \frac{9}{4} ; \\
\frac{3}{4} ; & x^{4}
\end{array}\right]+28 \eta x^{2}\left(1+x^{2}\right)^{\frac{7}{2}}{ }_{2} F_{1}\left[\begin{array}{lll}
\frac{5}{2}, & \frac{11}{4} ; & \\
\frac{5}{4} ; & & x^{4}
\end{array}\right] \\
& +10 \mu x^{3}\left(1+x^{2}\right)^{\frac{7}{2}}{ }_{2} F_{1}\left[\begin{array}{lll}
\frac{5}{2}, & \frac{15}{4} ; & \\
\frac{7}{4} ; & & x^{4}
\end{array}\right]+56 \eta x^{3}\left(1+x^{2}\right)^{\frac{5}{2}}{ }_{2} F_{1}\left[\begin{array}{lll}
\frac{5}{2}, & \frac{11}{4} ; & \\
\frac{5}{4} ; & & x^{4}
\end{array}\right]
\end{aligned}
$$

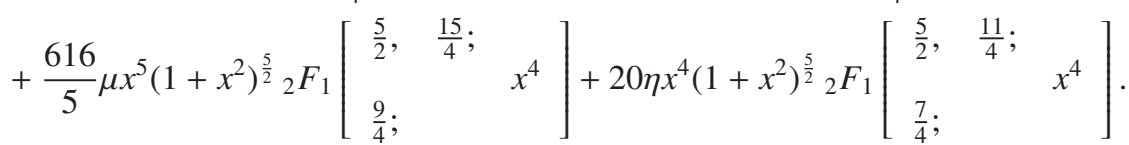

Proof. We shall derive only one result, say (26). Rest can be proved on similar lines. In order to derive (26), we proceed as follows. In (22), replace $x$ by $\frac{2 x}{1+x^{2}}$ and after some simplification, we have

$$
\begin{aligned}
{ }_{2} F_{1}\left[\begin{array}{rr}
\frac{1}{2}, & \frac{3}{2} ; \\
2 ; & \frac{1}{2}+\frac{x}{1+x^{2}}
\end{array}\right]= & 2 \mu_{2} F_{1}\left[\begin{array}{ll}
\frac{1}{4}, & \frac{5}{4} ; \\
\frac{1}{2} ; & \frac{4 x^{2}}{\left(1+x^{2}\right)^{2}}
\end{array}\right]-4 \eta_{2} F_{1}\left[\begin{array}{ll}
\frac{3}{4}, & \frac{3}{4} ; \\
\frac{1}{2} ; & \frac{4 x^{2}}{\left(1+x^{2}\right)^{2}}
\end{array}\right] \\
& -\frac{2 \mu x}{1+x^{2}}{ }_{2} F_{1}\left[\begin{array}{cc}
\frac{5}{4}, & \frac{5}{4} ; \\
\frac{3}{2} ; & \frac{4 x^{2}}{\left(1+x^{2}\right)^{2}}
\end{array}\right]+\frac{12 \eta x}{1+x^{2}}{ }_{2} F_{1}\left[\begin{array}{ccc}
\frac{3}{4}, & \frac{7}{4} ; \\
\frac{3}{2} ; & \frac{4 x^{2}}{\left(1+x^{2}\right)^{2}}
\end{array}\right] .
\end{aligned}
$$

On using appropriate results (8) and (11) for different values of $r$ and $m$ and after some algebra, we easily get the desired result (26). In exactly the same manner, other results (27) to (29) can be established.

\section{Results Closely Related to Ramanujan's Results (10)}

In this section, we shall establish the following interesting results closely related to the Ramanujan's result (10). These are

1)

$$
\begin{aligned}
& \left(1-x^{2}\right)^{\frac{3}{2}}{ }_{2} F_{1}\left[\begin{array}{ccc}
\frac{1}{2}, & \frac{3}{2} ; & \\
2 ; & \frac{1}{2}+\frac{x}{1+x^{2}}
\end{array}\right]=-4 \eta_{2} F_{1}\left[\begin{array}{ccc}
-\frac{1}{2}, & \frac{3}{2} ; & \\
\frac{1}{4} ; & & \frac{x^{4}}{x^{4}-1}
\end{array}\right]+2 \mu\left(1+x^{2}\right)_{2} F_{1}\left[\begin{array}{ccc}
-\frac{1}{2}, & \frac{3}{2} ; & \\
\frac{3}{4} ; & & \frac{x^{4}}{x^{4}-1}
\end{array}\right] \\
& +12 \eta x\left(1+x^{2}\right){ }_{2} F_{1}\left[\begin{array}{ccc}
-\frac{1}{2}, & \frac{3}{2} ; & \\
\frac{5}{4} ; & \frac{x^{4}}{x^{4}-1}
\end{array}\right]-2 \mu x_{2} F_{1}\left[\begin{array}{ccc}
-\frac{1}{2}, & \frac{3}{2} ; & \\
\frac{3}{4} ; & \frac{x^{4}}{x^{4}-1}
\end{array}\right] \\
& -12 \eta x^{2}{ }_{2} F_{1}\left[\begin{array}{ccc}
-\frac{1}{2}, & \frac{3}{2} ; & \\
\frac{5}{4} ; & & \frac{x^{4}}{x^{4}-1}
\end{array}\right]-\frac{10}{3} \mu x_{2}^{3} F_{1}\left[\begin{array}{ccc}
-\frac{1}{2}, & \frac{3}{2} ; & \\
\frac{7}{4} ; & & \frac{x^{4}}{x^{4}-1}
\end{array}\right] \text {. }
\end{aligned}
$$

2)

$$
\begin{aligned}
& \left(1-x^{2}\right)^{\frac{3}{2}}{ }_{2} F_{1}\left[\begin{array}{lll}
\frac{1}{2}, & \frac{3}{2} ; & \\
1 ; & \frac{1}{2}+\frac{x}{1+x^{2}}
\end{array}\right]=2 \eta_{2} F_{1}\left[\begin{array}{ccc}
-\frac{1}{2}, & \frac{3}{2} ; & \\
\frac{1}{4} ; & \frac{x^{4}}{x^{4}-1}
\end{array}\right]+\mu\left(1+x^{2}\right)_{2} F_{1}\left[\begin{array}{ccc}
-\frac{1}{2}, & \frac{3}{2} ; & \\
\frac{3}{4} ; & \frac{x^{4}}{x^{4}-1}
\end{array}\right] \\
& +6 \eta x\left(1+x^{2}\right)_{2} F_{1}\left[\begin{array}{cc}
-\frac{1}{2}, & \frac{3}{2} ; \\
\frac{5}{4} ; & \frac{x^{4}}{x^{4}-1}
\end{array}\right]+\mu x_{2} F_{1}\left[\begin{array}{ccc}
-\frac{1}{2}, & \frac{3}{2} ; \\
\frac{3}{4} ; & \frac{x^{4}}{x^{4}-1}
\end{array}\right] \\
& +6 \eta x_{2}^{2}{ }_{2} F_{1}\left[\begin{array}{cc}
-\frac{1}{2}, & \frac{3}{2} ; \\
\frac{5}{4} ; & \frac{x^{4}}{x^{4}-1}
\end{array}\right]+\frac{5}{3} \mu x_{2}^{3} F_{1}\left[\begin{array}{ccc}
-\frac{1}{2}, & \frac{3}{2} ; & \\
\frac{7}{4} ; & & \frac{x^{4}}{x^{4}-1}
\end{array}\right] \text {. }
\end{aligned}
$$


3)

$$
\begin{aligned}
& \left(1-x^{2}\right)^{\frac{5}{2}}{ }_{2} F_{1}\left[\begin{array}{lll}
\frac{1}{2}, & \frac{5}{2} ; \\
3 ; & \frac{1}{2}+\frac{x}{1+x^{2}}
\end{array}\right] \\
& =\frac{128}{3} \eta x\left(1-x^{4}\right){ }_{2} F_{1}\left[\begin{array}{cc}
-\frac{1}{2}, & \frac{3}{2} ; \\
\frac{5}{4} ; & \frac{x^{4}}{x^{4}-1}
\end{array}\right]+\frac{32}{9} \mu\left(1-x^{4}\right)_{2} F_{1}\left[\begin{array}{ccc}
-\frac{1}{2}, & \frac{3}{2} ; & \\
\frac{3}{4} ; & & \frac{x^{4}}{x^{4}-1}
\end{array}\right] \\
& -\frac{32}{3} \eta\left(1-x^{2}\right)\left(1+x^{2}\right)^{2}{ }_{2} F_{1}\left[\begin{array}{ccc}
-\frac{3}{2}, & \frac{5}{2} ; & \\
\frac{1}{4} ; & \frac{x^{4}}{x^{4}-1}
\end{array}\right]-\frac{224}{3} \eta x^{2}\left(1-x^{2}\right)\left(1+x^{2}\right)^{2}{ }_{2} F_{1}\left[\begin{array}{ccc}
-\frac{3}{2}, & \frac{5}{2} ; & \\
\frac{5}{4} ; & \frac{x^{4}}{x^{4}-1}
\end{array}\right] \\
& -\frac{80}{9} \mu x\left(1+x^{2}\right)_{2} F_{1}\left[\begin{array}{cc}
-\frac{3}{2}, & \frac{5}{2} ; \\
\frac{3}{4} ; & \frac{x^{4}}{x^{4}-1}
\end{array}\right]-\frac{80}{3} \mu x^{3}\left(1+x^{2}\right)_{2} F_{1}\left[\begin{array}{ccc}
-\frac{3}{2}, & \frac{5}{2} ; & \\
\frac{7}{4} ; & & \frac{x^{4}}{x^{4}-1}
\end{array}\right] \\
& +\frac{160}{9} \mu x^{2}{ }_{2} F_{1}\left[\begin{array}{cc}
-\frac{3}{2}, & \frac{5}{2} ; \\
\frac{3}{4} ; & \frac{x^{4}}{x^{4}-1}
\end{array}\right]+\frac{160}{3} \mu x^{4}{ }_{2} F_{1}\left[\begin{array}{ccc}
-\frac{3}{2}, & \frac{5}{2} ; & \\
\frac{7}{4} ; & & \frac{x^{4}}{x^{4}-1}
\end{array}\right]
\end{aligned}
$$

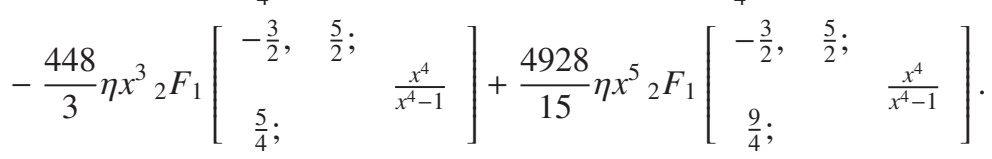

$$
\begin{aligned}
& \left(1-x^{2}\right)^{\frac{5}{2}}{ }_{2} F_{1}\left[\begin{array}{lll}
\frac{1}{2}, & \frac{5}{2} ; \\
1 ; & \frac{1}{2}+\frac{x}{1+x^{2}}
\end{array}\right] \\
& =\frac{4}{3} \mu x\left(1-x^{4}\right){ }_{2} F_{1}\left[\begin{array}{ccc}
-\frac{1}{2}, & \frac{3}{2} ; & \\
\frac{3}{4} ; & & \frac{x^{4}}{x^{4}-1}
\end{array}\right]+4 \eta\left(1+x^{2}\right)_{2} F_{1}\left[\begin{array}{ccc}
-\frac{3}{2}, & \frac{5}{2} ; & \\
\frac{1}{4} ; & & \frac{x^{4}}{x^{4}-1}
\end{array}\right] \\
& +16 \eta x\left(1-x^{4}\right)_{2} F_{1}\left[\begin{array}{ccc}
-\frac{1}{2}, & \frac{3}{2} ; & \\
\frac{5}{4} ; & & \frac{x^{4}}{x^{4}-1}
\end{array}\right]+\frac{10}{3} \mu x\left(1+x^{2}\right)_{2} F_{1}\left[\begin{array}{ccc}
-\frac{3}{2}, & \frac{5}{2} ; & \\
\frac{3}{4} ; & \frac{x^{4}}{x^{4}-1}
\end{array}\right] \\
& +\frac{20}{3} \mu x^{2}{ }_{2} F_{1}\left[\begin{array}{ccc}
-\frac{3}{2}, & \frac{5}{2} ; & \\
\frac{3}{4} ; & & \frac{x^{4}}{x^{4}-1}
\end{array}\right]+28 \eta x^{2}\left(1+x^{2}\right)_{2} F_{1}\left[\begin{array}{ccc}
-\frac{3}{2}, & \frac{5}{2} ; & \\
\frac{5}{4} ; & & \frac{x^{4}}{x^{4}-1}
\end{array}\right] \\
& +56 \eta x^{3}{ }_{2} F_{1}\left[\begin{array}{ccc}
-\frac{3}{2}, & \frac{5}{2} ; & \\
\frac{5}{4} ; & \frac{x^{4}}{x^{4}-1}
\end{array}\right]+10 \mu x^{3}\left(1+x^{2}\right)_{2} F_{1}\left[\begin{array}{ccc}
-\frac{3}{2}, & \frac{5}{2} ; & \\
\frac{7}{4} ; & & \frac{x^{4}}{x^{4}-1}
\end{array}\right]
\end{aligned}
$$

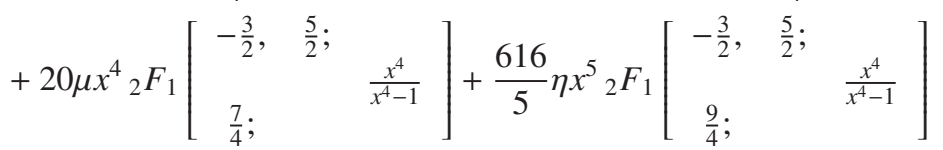

Proof. We shall derive only one of the results, say (30). Rest can be proved on similar lines. For this, if we apply Euler's transformation (4) in the each factors of ${ }_{2} F_{1}$ appearing on the right-hand side of (26), we have after some simplification arrive at the desired result (30). In exactly the same manner, the results (31) to (33) can be established with the help of the results (27) to (29) respectively by using Euler's transformation (4).

\section{New Hypergeometric Identities}

In this section, we shall establish several new identities. The 19 new hypergeometric identities given in the form of a single result to be established is 


$$
\begin{aligned}
& { } F_{2}\left[\begin{array}{cc}
a, \quad b, & e-d ; \\
\frac{1}{2}(a+b+i+1), \quad e ; & \left.\frac{1}{2}(1+x)\right] \\
= & \frac{\Gamma\left(\frac{1}{2}\right) \Gamma\left(\frac{1}{2} a+\frac{1}{2} b+\frac{1}{2} i+\frac{1}{2}\right) \Gamma\left(\frac{1}{2} a-\frac{1}{2} b-\frac{1}{2} i+\frac{1}{2}\right)}{\Gamma\left(\frac{1}{2} a-\frac{1}{2} b+\frac{1}{2}|i|+\frac{1}{2}\right)} \\
& \times\left[\sum_{j=0}^{\infty} \frac{\left(\frac{1}{2} a\right)_{j}\left(\frac{1}{2} a+\frac{1}{2}\right)_{j}\left(\frac{1}{2} b\right)_{j}\left(\frac{1}{2} b+\frac{1}{2}\right)_{j}}{\left(\frac{1}{2}\right)_{j} j !} \frac{\left(\frac{1}{2} d\right)_{j}\left(\frac{1}{2} d+\frac{1}{2}\right)_{j}}{\left(\frac{1}{2} e\right)_{j}\left(\frac{1}{2} e+\frac{1}{2}\right)_{j}}\right. \\
& \cdot\left\{\frac{C_{i}}{\Gamma\left(\frac{1}{2} a+\frac{1}{2}\right) \Gamma\left(\frac{1}{2} b+\frac{1}{2}+\frac{1}{2} i-\left[\frac{i+1}{2}\right]\right)\left(\frac{1}{2} a+\frac{1}{2}\right)_{j}\left(\frac{1}{2} b+\frac{1}{2}+\frac{1}{2} i-\left[\frac{i+1}{2}\right]\right)_{j}}\right. \\
& \left.+\frac{D_{i}}{\Gamma\left(\frac{1}{2} a\right) \Gamma\left(\frac{1}{2} b+\frac{1}{2} i-\left[\frac{i}{2}\right]\right)\left(\frac{1}{2} a\right)_{j}\left(\frac{1}{2} b+\frac{1}{2} i-\left[\frac{i}{2}\right]\right)_{j}}\right\} \\
& -\frac{a b d}{2 e} \sum_{j=0}^{\infty} \frac{\left(\frac{1}{2} a+\frac{1}{2}\right)_{j}\left(\frac{1}{2} a+1\right)_{j}\left(\frac{1}{2} b+\frac{1}{2}\right)_{j}\left(\frac{1}{2} b+1\right)_{j}}{\left(\frac{3}{2}\right)_{j} j !} \frac{\left(\frac{1}{2} d+\frac{1}{2}\right)_{j}\left(\frac{1}{2} d+1\right)_{j}}{\left(\frac{1}{2} e+\frac{1}{2}\right)_{j}\left(\frac{1}{2} e+1\right)_{j}} \\
& \cdot\left\{\frac{E_{i}}{\Gamma\left(\frac{1}{2} a+1\right) \Gamma\left(\frac{1}{2} b+\frac{1}{2} i+1-\left[\frac{i+1}{2}\right]\right)\left(\frac{1}{2} a+1\right)_{j}\left(\frac{1}{2} b+1+\frac{1}{2} i-\left[\frac{i+1}{2}\right]\right)_{j}}\right. \\
& \left.\left.+\frac{F_{i}}{\Gamma\left(\frac{1}{2} a+\frac{1}{2}\right) \Gamma\left(\frac{1}{2} b+\frac{1}{2}+\frac{1}{2} i-\left[\frac{i}{2}\right]\right)\left(\frac{1}{2} a+\frac{1}{2}\right)_{j}\left(\frac{1}{2} b+\frac{1}{2}+\frac{1}{2} i-\left[\frac{i}{2}\right]\right)_{j}}\right\}\right]
\end{array}\right.
\end{aligned}
$$

for $i=0, \pm 1, \ldots, \pm 9$.

Also, the coefficient $C_{i}$ and $D_{i}$ can be obtained from the Table 1 by changing $a$ to $a+2 j$ and $b$ to $b+2 j$ and the coefficients $E_{i}$ and $F_{i}$ can be obtained from Table 1 by changing $a$ to $a+2 j+1$ and $b$ to $b+2 j+1$ respectively.

Proof. In order to establish (34), we proceed as follows. First of all, changing $x$ to $-x$ in (16) and writing it in the following contracted notation

$$
\begin{aligned}
& { }_{2} F_{1}\left[\begin{array}{ccc}
a, & b, & \\
\frac{1}{2}(a+b+i+1), & & \frac{1-x}{2}
\end{array}\right]=\frac{\Gamma\left(\frac{1}{2}\right) \Gamma\left(\frac{1}{2} a+\frac{1}{2} b+\frac{1}{2} i+\frac{1}{2}\right) \Gamma\left(\frac{1}{2} a-\frac{1}{2} b-\frac{1}{2} i+\frac{1}{2}\right)}{\Gamma\left(\frac{1}{2} a-\frac{1}{2} b+\frac{1}{2}|i|+\frac{1}{2}\right)} \\
& \times\left\{\sum_{j=0}^{\infty} \alpha_{i, j} x^{2 j}+\frac{a b}{2} \sum_{j=0}^{\infty} \beta_{i, j} x^{2 j+1}\right\}
\end{aligned}
$$

where

$$
\begin{aligned}
\alpha_{i, j}= & \frac{\left(\frac{1}{2} a\right)_{j}\left(\frac{1}{2} a+\frac{1}{2}\right)_{j}\left(\frac{1}{2} b\right)_{j}\left(\frac{1}{2} b+\frac{1}{2}\right)_{j}}{\left(\frac{1}{2}\right)_{j} j !} \\
& \cdot\left\{\frac{C_{i}}{\Gamma\left(\frac{1}{2} a+\frac{1}{2}\right) \Gamma\left(\frac{1}{2} b+\frac{1}{2}+\frac{1}{2} i-\left[\frac{i+1}{2}\right]\right)\left(\frac{1}{2} a+\frac{1}{2}\right)_{j}\left(\frac{1}{2} b+\frac{1}{2}+\frac{1}{2} i-\left[\frac{i+1}{2}\right]\right)_{j}}\right. \\
& \left.+\frac{D_{i}}{\Gamma\left(\frac{1}{2} a\right) \Gamma\left(\frac{1}{2} b+\frac{1}{2} i-\left[\frac{i}{2}\right]\right)\left(\frac{1}{2} a\right)_{j}\left(\frac{1}{2} b+\frac{1}{2} i-\left[\frac{i}{2}\right]\right)_{j}}\right\}
\end{aligned}
$$

and 


$$
\begin{aligned}
\beta_{i, j}= & \frac{\left(\frac{1}{2} a+\frac{1}{2}\right)_{j}\left(\frac{1}{2} a+1\right)_{j}\left(\frac{1}{2} b\right)_{j}\left(\frac{1}{2} b+\frac{1}{2}\right)_{j}}{\left(\frac{3}{2}\right)_{j} j !} \\
& \cdot\left\{\frac{E_{i}}{\Gamma\left(\frac{1}{2} a+1\right) \Gamma\left(\frac{1}{2} b+\frac{1}{2} i+1-\left[\frac{i+1}{2}\right]\right)\left(\frac{1}{2} a+1\right)_{j}\left(\frac{1}{2} b+1+\frac{1}{2} i-\left[\frac{i+1}{2}\right]\right)_{j}}\right. \\
& \left.+\frac{F_{i}}{\Gamma\left(\frac{1}{2} a+\frac{1}{2}\right) \Gamma\left(\frac{1}{2} b+\frac{1}{2}+\frac{1}{2} i-\left[\frac{i}{2}\right]\right)\left(\frac{1}{2} a+\frac{1}{2}\right)_{j}\left(\frac{1}{2} b+\frac{1}{2}+\frac{1}{2} i-\left[\frac{i}{2}\right]\right)_{j}}\right\} .
\end{aligned}
$$

Now, multiplying both sides of (35) by $x^{d-1}(1-x)^{e-d-1}$ and integrating the resulting equation with respect to $x$ between the limit 0 to 1 , we have

$$
\begin{aligned}
& \int_{0}^{1} x^{d-1}(1-x)^{e-d-1}{ }_{2} F_{1}\left[\begin{array}{cc}
a, & b, \\
\frac{1}{2}(a+b+i+1), & \frac{1-x}{2}
\end{array}\right] d x \\
= & \frac{\Gamma\left(\frac{1}{2}\right) \Gamma\left(\frac{1}{2} a+\frac{1}{2} b+\frac{1}{2} i+\frac{1}{2}\right) \Gamma\left(\frac{1}{2} a-\frac{1}{2} b-\frac{1}{2} i+\frac{1}{2}\right)}{\Gamma\left(\frac{1}{2} a-\frac{1}{2} b+\frac{1}{2}|i|+\frac{1}{2}\right)} \\
& \times \int_{0}^{1} x^{d-1}(1-x)^{e-d-1}\left\{\sum_{j=0}^{\infty} \alpha_{i, j} x^{2 j}+\frac{a b}{2} \sum_{j=0}^{\infty} \beta_{i, j} x^{2 j+1}\right\} d x .
\end{aligned}
$$

Now, expressing ${ }_{2} F_{1}$ appearing on the left-hand side of (36), changing the order of integration and summation both sides, which is justified due to uniform convergence of the series involved in the process, we have after simplification

$$
\begin{aligned}
& \sum_{n=0}^{\infty} \frac{(a)_{n}(b)_{n}}{\left(\frac{1}{2}(a+b+i+1)\right)_{n} n !} \frac{1}{2^{n}} \int_{0}^{1} x^{d-1}(1-x)^{e-d+n-1} d x \\
= & \frac{\Gamma\left(\frac{1}{2}\right) \Gamma\left(\frac{1}{2} a+\frac{1}{2} b+\frac{1}{2} i+\frac{1}{2}\right) \Gamma\left(\frac{1}{2} a-\frac{1}{2} b-\frac{1}{2} i+\frac{1}{2}\right)}{\Gamma\left(\frac{1}{2} a-\frac{1}{2} b+\frac{1}{2}|i|+\frac{1}{2}\right)} \\
& \times \sum_{j=0}^{\infty} \alpha_{i, j} \int_{0}^{1} x^{d+2 j-1}(1-x)^{e-d-1}+\frac{a b}{2} \sum_{j=0}^{\infty} \beta_{i, j} \int_{0}^{1} x^{d+2 j}(1-x)^{e-d-1} d x .
\end{aligned}
$$

Now, evaluating the beta integral both sides and on the right-hand side using duplication formula for the gamma function and on the left-hand side summing up the series, after some simplification, we arrive at the desired result (34). This completes the proof of (34).

\subsection{Special Cases}

In this section, we shall consider some of the very interesting special cases of (34). These are

1) For $i=1$

$$
\begin{aligned}
{ }_{3} F_{2}\left[\begin{array}{ccc}
a, & b, & e-d ; \\
\frac{1}{2}(a+b+2), & e ;
\end{array}\right]= & \left(\frac{a+b}{a-b}\right) \Gamma\left(\frac{1}{2}\right) \Gamma\left(\frac{1}{2} a+\frac{1}{2} b\right) \\
& \times\left[\frac{1}{\Gamma\left(\frac{1}{2} a\right) \Gamma\left(\frac{1}{2} b+\frac{1}{2}\right)}{ }_{4} F_{3}\left[\begin{array}{cccc}
\frac{1}{2} a+\frac{1}{2}, & \frac{1}{2} b, & \frac{1}{2} d, & \frac{1}{2} d+\frac{1}{2} ; \\
\frac{1}{2}, & \frac{1}{2} e, & \frac{1}{2} e+\frac{1}{2} ; & 1
\end{array}\right]\right.
\end{aligned}
$$




$$
\begin{aligned}
& -\frac{1}{\Gamma\left(\frac{1}{2} a+\frac{1}{2}\right) \Gamma\left(\frac{1}{2} b\right)} 4 F_{3}\left[\begin{array}{ccccc}
\frac{1}{2} a, & \frac{1}{2} b+\frac{1}{2}, & \frac{1}{2} d, & \frac{1}{2} d+\frac{1}{2} ; & 1 \\
\frac{1}{2}, & \frac{1}{2} e, & \frac{1}{2} e+\frac{1}{2} ; &
\end{array}\right] \\
& +\frac{b d}{e} \frac{1}{\Gamma\left(\frac{1}{2} a\right) \Gamma\left(\frac{1}{2} b+\frac{1}{2}\right)}{ }_{4} F_{3}\left[\begin{array}{cccc}
\frac{1}{2} a+\frac{1}{2}, & \frac{1}{2} b+1, & \frac{1}{2} d+\frac{1}{2}, & \frac{1}{2} d+1 ; \\
\frac{3}{2}, & \frac{1}{2} e+\frac{1}{2}, & \frac{1}{2} e+1 ; & 1
\end{array}\right] \\
& -\frac{a d}{e} \frac{1}{\Gamma\left(\frac{1}{2} a+\frac{1}{2}\right) \Gamma\left(\frac{1}{2} b\right)}{ }_{4} F_{3}\left[\begin{array}{clll}
\frac{1}{2} a+1, & \frac{1}{2} b+\frac{1}{2}, & \frac{1}{2} d+\frac{1}{2}, & \frac{1}{2} d+1 ; \\
\frac{3}{2}, & \frac{1}{2} e+\frac{1}{2}, & \frac{1}{2} e+1 ; & 1
\end{array}\right] \text {. }
\end{aligned}
$$

Further, in this if we take $a=\frac{1}{2}, b=\frac{3}{2}$, we get

$$
\begin{aligned}
& { }_{3} F_{2}\left[\begin{array}{lll}
\frac{1}{2}, & \frac{3}{2}, & e-d ; \\
2, & e ; & \frac{1}{2}
\end{array}\right] \\
& =2 \mu_{4} F_{3}\left[\begin{array}{ccccc}
\frac{1}{4}, & \frac{5}{4}, & \frac{1}{2} d, & \frac{1}{2} d+\frac{1}{2} ; & 1 \\
\frac{1}{2}, & \frac{1}{2} e, & \frac{1}{2} e+\frac{1}{2} ; & 1
\end{array}\right]-4 \eta_{4} F_{3}\left[\begin{array}{ccccc}
\frac{3}{4}, & \frac{3}{4}, & \frac{1}{2} d, & \frac{1}{2} d+\frac{1}{2} ; & 1 \\
\frac{1}{2}, & \frac{1}{2} e, & \frac{1}{2} e+\frac{1}{2} ; &
\end{array}\right] \\
& +\frac{d}{e} \mu_{4} F_{3}\left[\begin{array}{cccc}
\frac{5}{4}, & \frac{5}{4}, & \frac{1}{2} d+\frac{1}{2}, & \frac{1}{2} d+1 ; \\
\frac{3}{2}, & \frac{1}{2} e+\frac{1}{2}, & \frac{1}{2} e+1 ; & 1
\end{array}\right]-6 \frac{d}{e} \mu_{4} F_{3}\left[\begin{array}{cccc}
\frac{3}{4}, & \frac{7}{4}, & \frac{1}{2} d+\frac{1}{2}, & \frac{1}{2} d+1 ; \\
\frac{3}{2}, & \frac{1}{2} e+\frac{1}{2}, & \frac{1}{2} e+1 ; & \\
\end{array}\right] .
\end{aligned}
$$

2) For $i=-1$

$$
\begin{aligned}
& { }_{3} F_{2}\left[\begin{array}{ccc}
a, & b, & e-d ; \\
\frac{1}{2}(a+b), & e ; & \frac{1}{2}
\end{array}\right]=\Gamma\left(\frac{1}{2}\right) \Gamma\left(\frac{1}{2} a+\frac{1}{2} b\right) \\
& \times\left[\frac{1}{\Gamma\left(\frac{1}{2} a+\frac{1}{2}\right) \Gamma\left(\frac{1}{2} b\right)}{ }_{4} F_{3}\left[\begin{array}{ccccc}
\frac{1}{2} a, & \frac{1}{2} b+\frac{1}{2}, & \frac{1}{2} d, & \frac{1}{2} d+\frac{1}{2} ; & 1 \\
\frac{1}{2}, & \frac{1}{2} e, & \frac{1}{2} e+\frac{1}{2} ; & & 1
\end{array}\right]\right. \\
& +\frac{1}{\Gamma\left(\frac{1}{2} a\right) \Gamma\left(\frac{1}{2} b+\frac{1}{2}\right)}{ }_{4} F_{3}\left[\begin{array}{ccccc}
\frac{1}{2} a+\frac{1}{2}, & \frac{1}{2} b, & \frac{1}{2} d, & \frac{1}{2} d+\frac{1}{2} ; & \\
\frac{1}{2}, & \frac{1}{2} e, & \frac{1}{2} e+\frac{1}{2} ; & & 1
\end{array}\right] \\
& -\frac{b d}{e} \frac{1}{\Gamma\left(\frac{1}{2} a\right) \Gamma\left(\frac{1}{2} b+\frac{1}{2}\right)}{ }_{4} F_{3}\left[\begin{array}{cccc}
\frac{1}{2} a+\frac{1}{2}, & \frac{1}{2} b+1, & \frac{1}{2} d+\frac{1}{2}, & \frac{1}{2} d+1 ; \\
\frac{3}{2}, & \frac{1}{2} e+\frac{1}{2}, & \frac{1}{2} e+1 ; & 1
\end{array}\right] \\
& -\frac{a d}{e} \frac{1}{\Gamma\left(\frac{1}{2} a+\frac{1}{2}\right) \Gamma\left(\frac{1}{2} b\right)}{ }_{4} F_{3}\left[\begin{array}{clll}
\frac{1}{2} a+1, & \frac{1}{2} b+\frac{1}{2}, & \frac{1}{2} d+\frac{1}{2}, & \frac{1}{2} d+1 ; \\
\frac{3}{2}, & \frac{1}{2} e+\frac{1}{2}, & \frac{1}{2} e+1 ; & 1
\end{array}\right] \text {. }
\end{aligned}
$$

Further, in this if we take $a=\frac{1}{2}, b=\frac{3}{2}$, we get

$$
\begin{aligned}
& { }_{3} F_{2}\left[\begin{array}{llll}
\frac{1}{2}, & \frac{3}{2}, & e-d ; & \\
2, & e ; & \frac{1}{2}
\end{array}\right] \\
& =\mu_{4} F_{3}\left[\begin{array}{ccccc}
\frac{1}{4}, & \frac{5}{4}, & \frac{1}{2} d, & \frac{1}{2} d+\frac{1}{2} ; & \\
\frac{1}{2}, & \frac{1}{2} e, & \frac{1}{2} e+\frac{1}{2} ; & 1
\end{array}\right]+2 \eta_{4} F_{3}\left[\begin{array}{cccc}
\frac{3}{4}, & \frac{3}{4}, & \frac{1}{2} d, & \frac{1}{2} d+\frac{1}{2} ; \\
\frac{1}{2}, & \frac{1}{2} e, & \frac{1}{2} e+\frac{1}{2} ; &
\end{array}\right] \\
& -\frac{d}{2 e} \mu_{4} F_{3}\left[\begin{array}{cccc}
\frac{5}{4}, & \frac{5}{4}, & \frac{1}{2} d+\frac{1}{2}, & \frac{1}{2} d+1 ; \\
\frac{3}{2}, & \frac{1}{2} e+\frac{1}{2}, & \frac{1}{2} e+1 ; & 1
\end{array}\right]-3 \frac{d}{e} \eta_{4} F_{3}\left[\begin{array}{cccc}
\frac{3}{4}, & \frac{7}{4}, & \frac{1}{2} d+\frac{1}{2}, & \frac{1}{2} d+1 ; \\
\frac{3}{2}, & \frac{1}{2} e+\frac{1}{2}, & \frac{1}{2} e+1 ; & 1
\end{array}\right] \text {. }
\end{aligned}
$$


3) For $i=2$

$$
\begin{aligned}
& { }_{3} F_{2}\left[\begin{array}{cccc}
a, & b, & e-d ; & \frac{1}{2} \\
\frac{1}{2}(a+b+3), & e ; &
\end{array}\right] \\
& =\frac{\Gamma\left(\frac{1}{2}\right) \Gamma\left(\frac{1}{2} a+\frac{1}{2} b+\frac{3}{2}\right) \Gamma\left(\frac{1}{2} a-\frac{1}{2} b-\frac{1}{2}\right)}{\Gamma\left(\frac{1}{2} a-\frac{1}{2} b+\frac{3}{2}\right)} \\
& \times\left[\frac{(b+a-1)}{2 \Gamma\left(\frac{1}{2} a+\frac{1}{2}\right) \Gamma\left(\frac{1}{2} b+\frac{1}{2}\right)}{ }_{4} F_{3}\left[\begin{array}{cllll}
\frac{1}{2} a, & \frac{1}{2} b, & \frac{1}{2} d, & \frac{1}{2} d+\frac{1}{2} ; & 1 \\
\frac{1}{2}, & \frac{1}{2} e, & \frac{1}{2} e+\frac{1}{2} ; &
\end{array}\right]\right. \\
& -\frac{2}{\Gamma\left(\frac{1}{2} a\right) \Gamma\left(\frac{1}{2} b\right)}{ }_{4} F_{3}\left[\begin{array}{ccccc}
\frac{1}{2} a+\frac{1}{2}, & \frac{1}{2} b+\frac{1}{2}, & \frac{1}{2} d, & \frac{1}{2} d+\frac{1}{2} ; & \\
\frac{1}{2}, & \frac{1}{2} e, & \frac{1}{2} e+\frac{1}{2} ; & & 1
\end{array}\right] \\
& -\frac{d}{e} \frac{(a+b+1)}{\Gamma\left(\frac{1}{2} a\right) \Gamma\left(\frac{1}{2} b\right)}{ }_{4} F_{3}\left[\begin{array}{cccc}
\frac{1}{2} a+\frac{1}{2}, & \frac{1}{2} b+\frac{1}{2}, & \frac{1}{2} d+\frac{1}{2}, & \frac{1}{2} d+1 ; \\
\frac{3}{2}, & \frac{1}{2} e+\frac{1}{2}, & \frac{1}{2} e+1 ; & 1
\end{array}\right] \\
& +\frac{d}{e} \frac{a b}{\Gamma\left(\frac{1}{2} a+\frac{1}{2}\right) \Gamma\left(\frac{1}{2} b+\frac{1}{2}\right)}{ }_{4} F_{3}\left[\begin{array}{cccc}
\frac{1}{2} a+1, & \frac{1}{2} b+1, & \frac{1}{2} d+\frac{1}{2}, & \frac{1}{2} d+1 ; \\
\frac{3}{2}, & \frac{1}{2} e+\frac{1}{2}, & \frac{1}{2} e+1 ; & 1
\end{array}\right] \\
& +\frac{d(d+1)}{e(e+1)} \frac{a b}{\Gamma\left(\frac{1}{2} a+\frac{1}{2}\right) \Gamma\left(\frac{1}{2} b+\frac{1}{2}\right)}{ }_{4} F_{3}\left[\begin{array}{cccc}
\frac{1}{2} a+1, & \frac{1}{2} b+1, & \frac{1}{2} d+1, & \frac{1}{2} d+\frac{3}{2} ; \\
\frac{3}{2}, & \frac{1}{2} e+1, & \frac{1}{2} e+\frac{3}{2} ; & 1
\end{array}\right] \\
& \left.-\frac{2}{3} \frac{d(d+1)(d+2)}{e(e+1)(e+2)} \frac{(a+1)(b+1)}{\Gamma\left(\frac{1}{2} a\right) \Gamma\left(\frac{1}{2} b\right)}{ }_{4} F_{3}\left[\begin{array}{cccc}
\frac{1}{2} a+\frac{3}{2}, & \frac{1}{2} b+\frac{3}{2}, & \frac{1}{2} d+\frac{3}{2}, & \frac{1}{2} d+2 ; \\
\frac{5}{2}, & \frac{1}{2} e+\frac{3}{2}, & \frac{1}{2} e+2 ; & 1
\end{array}\right]\right] .
\end{aligned}
$$

Further, in this if we take $a=\frac{1}{2}, b=\frac{5}{2}$, we get

$$
\begin{aligned}
& { }_{3} F_{2}\left[\begin{array}{cccc}
\frac{1}{2}, & \frac{5}{2}, \quad e-d ; \\
3, & e ; & \frac{1}{2}
\end{array}\right] \\
& =\frac{32}{9} \mu_{4} F_{3}\left[\begin{array}{ccccc}
\frac{1}{4}, & \frac{5}{4}, & \frac{1}{2} d, & \frac{1}{2} d+\frac{1}{2} ; & 1 \\
\frac{1}{2}, & \frac{1}{2} e, & \frac{1}{2} e+\frac{1}{2} ; &
\end{array}\right] \\
& -\frac{32}{3} \eta_{4} F_{3}\left[\begin{array}{ccccc}
\frac{3}{4}, & \frac{7}{4}, & \frac{1}{2} d, & \frac{1}{2} d+\frac{1}{2} ; & 1 \\
\frac{1}{2}, & \frac{1}{2} e, & \frac{1}{2} e+\frac{1}{2} ; & & 1
\end{array}\right] \\
& -\frac{64}{3} \eta\left(\frac{d}{e}\right){ }_{4} F_{3}\left[\begin{array}{cccc}
\frac{3}{4}, & \frac{7}{4}, & \frac{1}{2} d+\frac{1}{2}, & \frac{1}{2} d+1 ; \\
\frac{3}{2}, & \frac{1}{2} e+\frac{1}{2}, & \frac{1}{2} e+1 ; & 1
\end{array}\right] \\
& +\frac{40}{9} \mu\left(\frac{d}{e}\right){ }_{4} F_{3}\left[\begin{array}{cccc}
\frac{5}{4}, & \frac{9}{4}, & \frac{1}{2} d+\frac{1}{2}, & \frac{1}{2} d+1 ; \\
\frac{3}{2}, & \frac{1}{2} e+\frac{1}{2}, & \frac{1}{2} e+1 ; & 1
\end{array}\right] \\
& +\frac{40}{9} \mu\left(\frac{d(d+1)}{e(e+1)}\right){ }_{4} F_{3}\left[\begin{array}{cccc}
\frac{5}{4}, & \frac{9}{4}, & \frac{1}{2} d+1, & \frac{1}{2} d+\frac{3}{2} ; \\
\frac{3}{2}, & \frac{1}{2} e+1, & \frac{1}{2} e+\frac{3}{2} ; & 1
\end{array}\right] \\
& -\frac{56}{3} \eta\left(\frac{d(d+1)(d+2)}{e(e+1)(e+2)}\right){ }_{4} F_{3}\left[\begin{array}{ccccc}
\frac{7}{4}, & \frac{11}{4}, & \frac{1}{2} d+\frac{3}{2}, & \frac{1}{2} d+2 ; & \\
\frac{5}{2}, & \frac{1}{2} e+\frac{3}{2}, & \frac{1}{2} e+2 ; & 1
\end{array}\right] .
\end{aligned}
$$


4) For $i=-2$

$$
\begin{aligned}
& { }_{3} F_{2}\left[\begin{array}{cccc}
a, & b, & e-d ; & \\
\frac{1}{2}(a+b-1), & e ; &
\end{array}\right] \\
& =\Gamma\left(\frac{1}{2}\right) \Gamma\left(\frac{1}{2} a+\frac{1}{2} b-\frac{1}{2}\right) \\
& \times\left[\frac{(b+a-1)}{2 \Gamma\left(\frac{1}{2} a+\frac{1}{2}\right) \Gamma\left(\frac{1}{2} b+\frac{1}{2}(())\right)}{ }_{4} F_{3}\left[\begin{array}{ccccc}
\frac{1}{2} a, & \frac{1}{2} b, & \frac{1}{2} d, & \frac{1}{2} d+\frac{1}{2} ; & \\
\frac{1}{2}, & \frac{1}{2} e, & \frac{1}{2} e+\frac{1}{2} ; &
\end{array}\right]\right. \\
& +\frac{2}{\Gamma\left(\frac{1}{2} a\right) \Gamma\left(\frac{1}{2} b\right)}{ }_{4} F_{3}\left[\begin{array}{ccccc}
\frac{1}{2} a+\frac{1}{2}, & \frac{1}{2} b+\frac{1}{2}, & \frac{1}{2} d, & \frac{1}{2} d+\frac{1}{2} ; & \\
\frac{1}{2}, & \frac{1}{2} e, & \frac{1}{2} e+\frac{1}{2} ; & & 1
\end{array}\right] \\
& -\frac{a b}{\Gamma\left(\frac{1}{2} a+\frac{1}{2}\right) \Gamma\left(\frac{1}{2} b+\frac{1}{2}\right)}\left(\frac{d}{e}\right){ }_{4} F_{3}\left[\begin{array}{cccc}
\frac{1}{2} a+1, & \frac{1}{2} b+1, & \frac{1}{2} d+\frac{1}{2}, & \frac{1}{2} d+1 ; \\
\frac{3}{2}, & \frac{1}{2} e+\frac{1}{2}, & \frac{1}{2} e+1 ; & 1
\end{array}\right] \\
& -\frac{a+b+1}{\Gamma\left(\frac{1}{2} a\right) \Gamma\left(\frac{1}{2} b\right)}\left(\frac{d}{e}\right)_{4} F_{3}\left[\begin{array}{cccc}
\frac{1}{2} a+\frac{1}{2}, & \frac{1}{2} b+\frac{1}{2}, & \frac{1}{2} d+\frac{1}{2}, & \frac{1}{2} d+1 ; \\
\frac{3}{2}, & \frac{1}{2} e+\frac{1}{2}, & \frac{1}{2} e+1 ; & 1
\end{array}\right] \\
& +\frac{d(d+1)}{e(e+1)} \frac{a b}{\Gamma\left(\frac{1}{2} a+\frac{1}{2}\right) \Gamma\left(\frac{1}{2} b+\frac{1}{2}\right)}{ }_{4} F_{3}\left[\begin{array}{cccc}
\frac{1}{2} a+1, & \frac{1}{2} b+1, & \frac{1}{2} d+1, & \frac{1}{2} d+\frac{3}{2} ; \\
\frac{3}{2}, & \frac{1}{2} e+1, & \frac{1}{2} e+\frac{3}{2} ; & 1
\end{array}\right] \\
& -\frac{2}{3} \frac{d(d+1)(d+2)}{e(e+1)(e+2)} \frac{(a+1)(b+1)}{\Gamma\left(\frac{1}{2} a+\frac{1}{2}\right) \Gamma\left(\frac{1}{2} b+\frac{1}{2}\right)}{ }_{4} F_{3}\left[\begin{array}{clll}
\frac{1}{2} a+\frac{3}{2}, & \frac{1}{2} b+\frac{3}{2}, & \frac{1}{2} d+\frac{3}{2}, & \frac{1}{2} d+2 ; \\
\frac{5}{2}, & \frac{1}{2} e+\frac{3}{2}, & \frac{1}{2} e+2 ; & 1
\end{array}\right] \text {. }
\end{aligned}
$$

Further, in (43) if we take $a=\frac{1}{2}, b=\frac{5}{2}$, we obtain

$$
\begin{aligned}
& { }_{3} F_{2}\left[\begin{array}{llll}
\frac{1}{2}, & \frac{5}{2}, & e-d ; & \\
1, & e ; & \frac{1}{2}
\end{array}\right] \\
& =\frac{4}{3} \mu_{4} F_{3}\left[\begin{array}{ccccc}
\frac{1}{4}, & \frac{5}{4}, & \frac{1}{2} d, & \frac{1}{2} d+\frac{1}{2} ; & \\
\frac{1}{2}, & \frac{1}{2} e, & \frac{1}{2} e+\frac{1}{2} ; & & 1
\end{array}\right] \\
& +4 \eta_{4} F_{3}\left[\begin{array}{ccccc}
\frac{3}{4}, & \frac{7}{4}, & \frac{1}{2} d, & \frac{1}{2} d+\frac{1}{2} ; & \\
\frac{1}{2}, & \frac{1}{2} e, & \frac{1}{2} e+\frac{1}{2} ; & 1
\end{array}\right] \\
& -\frac{5}{3} \mu\left(\frac{d}{e}\right){ }_{4} F_{3}\left[\begin{array}{ccccc}
\frac{5}{4}, & \frac{9}{4}, & \frac{1}{2} d+\frac{1}{2}, & \frac{1}{2} d+1 ; & \\
\frac{3}{2}, & \frac{1}{2} e+\frac{1}{2}, & \frac{1}{2} e+1 ; & 1
\end{array}\right] \\
& -8 \eta\left(\frac{d}{e}\right){ }_{4} F_{3}\left[\begin{array}{ccccc}
\frac{3}{4}, & \frac{7}{4}, & \frac{1}{2} d+\frac{1}{2}, & \frac{1}{2} d+1 ; \\
\frac{3}{2}, & \frac{1}{2} e+\frac{1}{2}, & \frac{1}{2} e+1 ; & 1
\end{array}\right] \\
& +\frac{5}{3} \mu\left(\frac{d(d+1)}{e(e+1)}\right){ }_{4} F_{3}\left[\begin{array}{cccc}
\frac{5}{4}, & \frac{9}{4}, & \frac{1}{2} d+1, & \frac{1}{2} d+\frac{3}{2} ; \\
\frac{3}{2}, & \frac{1}{2} e+1, & \frac{1}{2} e+\frac{3}{2} ; & 1
\end{array}\right] \\
& +7 \eta\left(\frac{d(d+1)(d+2)}{e(e+1)(e+2)}\right)_{4} F_{3}\left[\begin{array}{ccccc}
\frac{7}{4}, & \frac{11}{4}, & \frac{1}{2} d+\frac{3}{2}, & \frac{1}{2} d+2 ; & \\
\frac{5}{2}, & \frac{1}{2} e+\frac{3}{2}, & \frac{1}{2} e+2 ; & & 1
\end{array}\right] .
\end{aligned}
$$

Similarly other results can also be obtained. 
We conclude this section by remarking that the results (37), (39), (41) and (43) are closely related to the Krattenthaler an Rao's result (12) while the results (38), (40), (42) and (44) are closely related to (13). The results (37) to (40) are also recorded in (Choi et al., 2013).

\section{Acknowledgements}

(1) The authors are thankful to the referees for making certain very useful suggestions.

(2) All authors contributed equally in this paper. They read and approved the final manuscript.

\section{References}

Bailey, W. N. (1964). Generalized hypergeometric series, Cambridge tracts in Mathematics and Mathematical Physics, 32. Cambridge, London and New York: Cambridge University Press. Reprinted by Stechert-Hafner Service Agency, New York and London.

Berndt, B. C. (1989). Ramanujan's Notebooks (Part II). Berlin, Heidelberg and New York: Springer-Verlage.

Choi, J., Rathie, A. K., \& Srivastava, H. M. (2011). A generalization of a formula due to Kummer. Integral Transform and Special Functions, 22(11), 881-889. http://dx.doi.org/10.1080/10652469.2011.588786

Choi, J., Rathie, A. K., \& Srivastava, H. M. (2013). Certain hypergeometric identities deducible using Beta integral method. Bull. Korean Math. Soc., 50(5), 1673-1681. http://dx.doi.org/10.4134/BKMS.2013.50.5.1673

Gauss, C. F. (1813). Disquisitiones generals circa seriem infinitam $1+\frac{\alpha \cdot \beta}{1 \cdot \gamma} x+\frac{\alpha(\alpha+1) \cdot \beta(\beta+1)}{1 \cdot 2 \cdot \gamma(\gamma+1)} x^{2}+\ldots$ (Göttingen Thesis 1812). Comment Soc. Regia Sci. Göttingen, pp. 3-46. Reprinted in Carl Friedrich Gauss Werke (12 Vols), Vol. 3, pp. 123-162 (see also pp. 197-202 and pp. 207-230), Göttingen, 1870-1933.

Kim, Y. S., Rakha, M. A., \& Rathie, A. K. (2010). Extensions of certain classical summation theorems for the series ${ }_{2} F_{1},{ }_{3} F_{2}$ and ${ }_{4} F_{3}$ with applications in Ramanujan's summation. Int. J. Math. \& Math. Sci., ID 309503, 26 pages. http://dx.doi.org/10.1155/2010/309503

Krattenthaler, C., \& Rao, K. S. (2003). Automatic generation of hypergeometric identities by the beta integral method. J. of Comput. Appl. Math., 160, 159-173. http://dx.doi.org/10.1016/S0377-0427(03)00629-0

Kummer, E. E. (1836). Uber die hypergeometrische Reihe $1+\frac{\alpha \cdot \beta}{1 \cdot \gamma} x+\frac{\alpha(\alpha+1) \cdot \beta(\beta+1)}{1 \cdot 2 \cdot \gamma(\gamma+1)} x^{2}+\ldots$ J. Reine Angew. Math. 15, pp. 39-83 and 127-172; see also, Collected papers, Vol. II: Function Theory, Geometry and Miscellaneous (Edited and with a forward by Andre Weil). Springer-Verlag, Berlin, Heidelberg and New York (1975).

Lavoie, J. L., Grondin, F., \& Rathie, A. K. (1992). Generalizations of Watson's theorem on the sum of a ${ }_{3} F_{2}$. Indian J. Math., 34, 23-32.

Lavoie, J. L., Grondin, F., Rathie, A. K., \& Arora, K. (1994). Generalizations of Dixon's theorem on the sum of a ${ }_{3} F_{2}$. Math. Comput.,62, 267-276.

Lavoie, J. L., Grondin, F., \& Rathie, A. K. (1996). Generalizations of Whipple's theorem on the sum of a ${ }_{3} F_{2} . J$. Comput. Appl. Math., 72, 293-300. http://dx.doi.org/10.1016/0377-0427(95)00279-0

Rakha, M. A., Pandey, U., \& Rathie, A. K. (2014). Some applications of generalized Kummer's summation theorem for the series ${ }_{2} F_{1}$. Submitted for Publication.

Rakha, M. A., Ibrahim, A. K., \& Rathie, A. K. (2013). Remark on two results due to Ramanujan on hypergeometric series. Arxive: 1302.4902v1 [math.cv].

Rakha, M. A., \& Rathie, A. K. (2011). Generalization of classical summation theorems of the series ${ }_{2} F_{1}$ and ${ }_{3} F_{2}$ with applications. Integral Transforms and Special Functions, 22(11), 823-840. http://dx.doi.org/10.1080/10652469.2010.549487

Rainville, E. D. (1960). Special Functions. New York: The Macmillan Company. Reprinted by Chelsea Publishing, Bronx, New York (1971).

Ramanujan, S. (1957). Notebooks (Vol. 1, 2). Tata Institute of Fundamental Research, Bombay.

Rathie, A. K., \& Kim, Y. S. (2001). On two results contiguous to a quadratic transformation due to Gauss. Far East J. Math. Sci., 31(1), 51-58.

Srivastava, H. M., \& Karlsson, P. W. (1985). Multiple Gaussian Hypergeometric Series (Ellis Horwood Limited, Chichester). New York, Chichester: John Wiley and Sons. 


\section{Copyrights}

Copyright for this article is retained by the author(s), with first publication rights granted to the journal.

This is an open-access article distributed under the terms and conditions of the Creative Commons Attribution license (http://creativecommons.org/licenses/by/3.0/). 